\title{
Rapidly Evolving Treatment Landscape for Metastatic Esophagogastric Carcinoma: Review of Recent Data
}

Lionel Aurelien Kankeu Fonkoua (D) ${ }^{1,2}$ Harry H Yoon'

'Department of Oncology, Mayo Clinic, Rochester, MN, 55905, USA;

${ }^{2}$ Department of Hematology, Mayo

Clinic, Rochester, MN, 55905, USA
Correspondence: Harry $\mathrm{H}$ Yoon Mayo Clinic, 200 First Street SW, Rochester, MN, 55905, USA

Email Yoon.Harry@mayo.edu

\begin{abstract}
Esophagogastric cancer (EGC) is a heterogeneous group of malignancies that collectively represent the 2 nd leading cause of cancer deaths worldwide. While surgery in combination with chemotherapy and/or radiation therapy represents the primary curative treatment for early stage disease, survival outcomes for the majority of patients with laterstage disease remain poor. Cytotoxic chemotherapy with platinum doublets such as 5-FU/ leucovorin/oxaliplatin is the mainstay of treatment with incremental benefits provided by targeted therapy (trastuzumab, trastuzumab deruxtecan, ramucirumab) and immunotherapy (pembrolizumab, nivolumab). In this article, we provide an updated review and perspectives on the management of advanced EGC. We examine the distinct epidemiological, etiological and molecular features of each disease entity comprising EGC. After reviewing the critical studies that established conventional systemic cytotoxic and targeted therapeutics, we elaborate on recent promising and complex data with immune checkpoint inhibition focusing on implications of tumor histology and PD-L1 expression in the tumor microenvironment. We also highlight novel diagnostic and therapeutic strategies to build on these recent advances. Keywords: esophagogastric cancer, gastric cancer, esophageal cancer, targeted therapy, immunotherapy, precision medicine
\end{abstract}

\section{Introduction}

Esophagogastric cancer (EGC) is a heterogeneous group of malignancies which, for practical reasons, are often divided into three disease entities: esophageal squamous cell carcinoma (ESCC) typically in the mid-to-proximal esophagus, gastroesophageal junction (GEJ) adenocarcinomas which originate in the distal esophagus or gastric cardia, and gastric carcinoma (GC) arising in the sub-cardia stomach. Although their estimated annual incidence in the United States (US) is only about 45,000 cases and 27,000 deaths, worldwide they collectively represent the 2nd leading cause of cancer deaths with the highest incidence seen in Eastern Asia and Central and Eastern Europe. ${ }^{1,2}$ Surgical resection in combination with chemotherapy and/or radiation therapy represents the primary curative treatment for early stage disease, but for the unfortunate majority of patients with unresectable or metastatic disease at the time of diagnosis, outcomes with palliative treatment remain poor with a 5-year survival of rate of about $5 \%{ }^{3}$ Cytotoxic chemotherapy is the mainstay of treatment with incremental benefits provided by targeted therapeutics against the human epidermal growth factor receptor 2 (HER2) and vascular 
endothelial growth factor receptor 2 (VEGFR-2). More recently, the emergence of immunotherapy into the treatment landscape of EGC is rapidly changing treatment paradigms and providing opportunities for combinatorial strategies.

In this article, we provide an updated review and perspectives on the management of refractory advanced EGC. We examine the distinct epidemiological, etiological and molecular features of each disease entity comprising EGC. We then highlight the critical studies that established conventional systemic cytotoxic and targeted therapeutics, and elaborate on recent promising data with immune checkpoint inhibition.

\section{Epidemiology and Etiological Risk Factors}

The anatomical, histopathological and geographical variation in EGC incidence is a reflection of the differing risk factors and tumorigenesis of the affected populations. ESCC representing about $90 \%$ of esophageal cancers worldwide occurs predominantly in Asia, Africa and South America, and often develops in the setting of tobacco and alcohol use. ${ }^{4-6}$ In contrast, esophageal adenocarcinoma (EAC) is more common in Western Europe and North America, often develops in the setting of smoking, obesity, GERD and Barrett's esophagus. ${ }^{7,8}$ While its incidence has dramatically risen over the past several decades in parallel with changes in the dietary and lifestyle habits of the Western world, the incidence of GC has declined in most age groups (but not the young), potentially due in part to the identification of the role of $H$. pylori in gastric carcinogenesis and implementation of population-based screening programs, food refrigeration and improved sanitation. ${ }^{9-12}$ Preliminary data also suggests that human papilloma virus (HPV) infection may play a role in both ESCC and EAC, although this remains under investigation. $^{13,14}$

\section{Molecular Characterization of EGC}

Advances in next-generation sequencing (NGS) technologies and molecular profiling have facilitated the characterization of the genomic landscape of EGC. The Cancer Genome Atlas (TCGA) Research Network integrated the results of a wide-scale molecular analysis of GC into a new classification system encompassing four molecular subtypes with distinct salient genomic features (Figure 1): ${ }^{15}$

- Epstein-Barr virus-positive (EBV; 9\%)
- Microsatellite instability (MSI; 22\%)

- Chromosomal instability (CIN; 50\%)

- Genomically stable (GS; 20\%)

The EBV-positive tumors were predominantly located in the gastric fundus or body, and exhibited higher prevalence of DNA promoter hypermethylation, $\mathrm{A}$ to $\mathrm{C}$ transversions, PIK3CA mutation, recurrent JAK2 and $E R B B 2$ amplifications. Additionally, the presence of viral antigens, strong interleukin-12 (IL-12) mediated signaling and PD-L1/2 overexpression suggests a robust immune cell presence, and provides support for targeted immunotherapy. The MSI tumors lacked targetable amplifications but were characterized by genomic instability due to a deficient DNA mismatch repair system and high rate of PD-L1 expression. The CIN tumors were more frequent in the GEJ/cardia and exhibited the highest frequency of TP53 mutations (71\%), as well as genomic amplifications of receptor tyrosine kinases (RTKs) and cell cycle mediators. The GS tumors located in the antrum exhibited elevated expression of molecules in the cell adhesion and angiogenesis-related pathways.

In the esophagus, the TCGA identified three molecular subtypes of ESCC (ESCC1-3). ${ }^{16}$ ESCC1 was characterized by alterations in the $N R F 2$ pathway and a higher frequency of SOX2 and/or TP63 amplification. ESCC2 exhibited higher rates of mutation of NOTCH1 or ZNF750 and greater leukocyte infiltration, while ESCC3 had sustained alterations predicted to activate the PI3K pathway. As expected, the squamous and adenocarcinoma histologies were molecularly distinct, with ESCC exhibiting frequent genomic amplifications of CCND1 and SOX2 and/or TP63, whereas EAC resembled the CIN variant of $\mathrm{GC}$ and displayed DNA hypermethylation and amplification of ERBB2, VEGFA, GATA4, and GATA6.

Together, these new molecular classifications are complementary to conventional subtyping based on histopathology and provide new insights into the tumor heterogeneity of EGC. Importantly, these data are expected to facilitate the identification of therapeutic targets and predictive biomarkers, as well as improve patient stratification for clinical trial design.

\section{Cytotoxic Chemotherapy}

For the majority of EGC patients ineligible for potentially curative therapies, palliative chemotherapy remains the cornerstone of management, with targeted therapy providing additional benefit. ${ }^{17}$ Combination chemotherapy has 


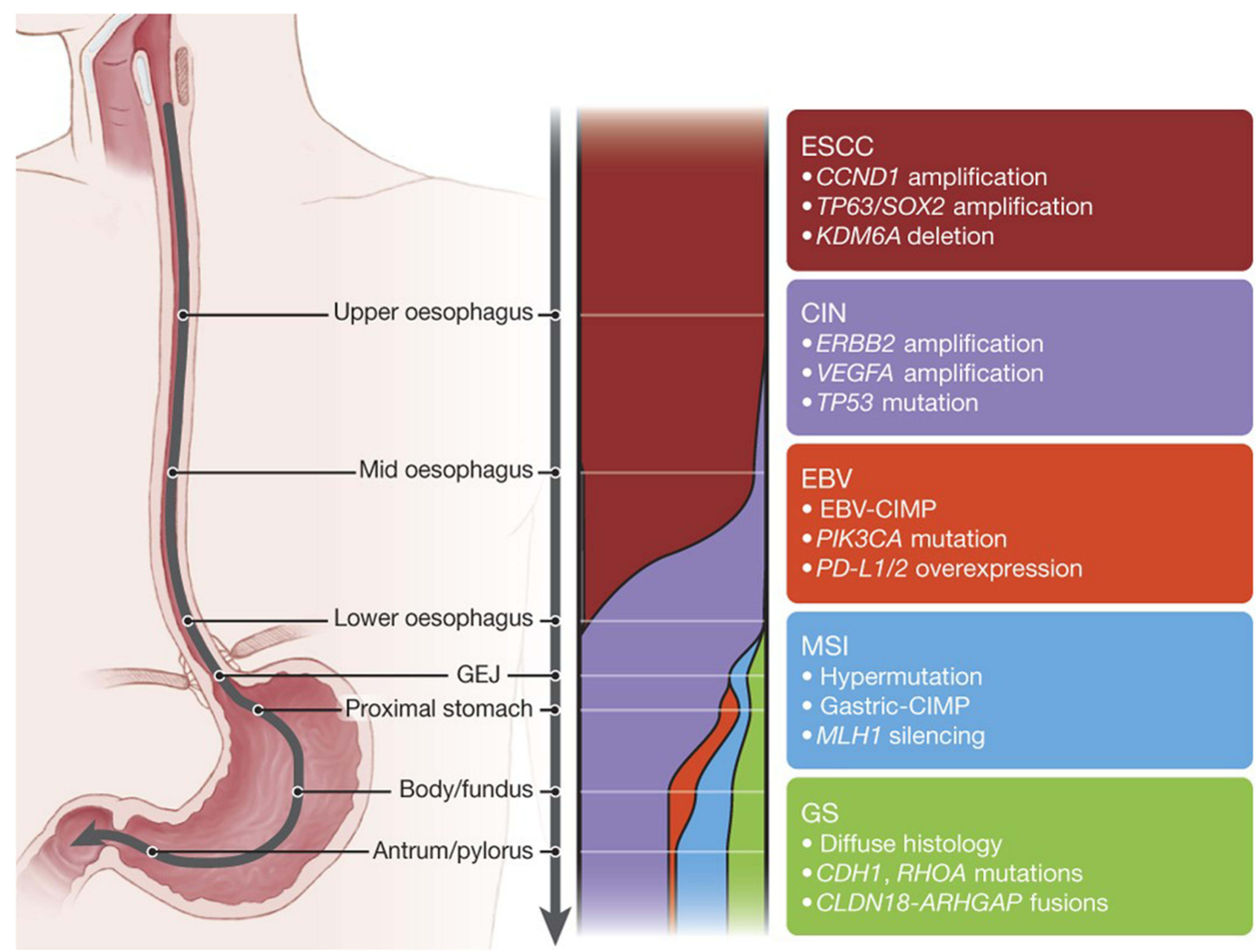

Figure I The four molecular subtypes of esophagogastric cancer described in the TCGA study, their mutational patterns, and location. Note:Reproduced from Cancer Genome Atlas Research Network. Integrated genomic characterization of oesophageal carcinoma. Nature. 2017;54I (7636): 169. doi:I0.1038/nature20805. ${ }^{16}$

Abbreviations: CIN, chromosomal instability; EBV, Epstein-Barr virus; GE, gastroesophageal junction; GS, genomically stable; MSI, microsatellite instability.

been proven to be more effective than single-agent chemotherapy, with the most rigorously evaluated regimens consisting of anthracycline, fluoropyrimidine, taxane, and platinum-based agents. In the first-line setting, platinum doublets such as 5-FU/leucovorin/oxaliplatin (eg, FLO or FOLFOX) have been established as preferred for advanced HER2-negative disease based on their toxicity profile and efficacy data (mOS 10.7 mos; mPFS 5.8 mos; ORR $34 \%) .{ }^{18}$ Anthracycline triplet combinations including epirubicin, oxaliplatin, and capecitabine (EOX) demonstrate comparable efficacy (mOS 11.2 mos; mPFS 7 mos; ORR $47.9 \%),{ }^{18}$ but remain less favored in the US due to questions on whether the anthracycline adds benefit, although the two types of regimens have never been compared head-to-head. ${ }^{19}$ Efficacy of first-line therapy was further improved in the HER2-positive population by the addition of trastuzumab to cisplatin/5-FU (mOS 13.8 mos; mPFS $6.5 \mathrm{mos} ;$ ORR 47\%). ${ }^{20} \quad$ 5-FU/leucovorin/irinotecan (FOLFIRI) remains a viable first-line option for patients who cannot receive platinum agents. ${ }^{21,22}$

In the second-line setting, ramucirumab alone or combined with paclitaxel is a standard option for adenocarcinomas, based on data from the REGARD and RAINBOW Phase 3 studies (discussed later in the targeted therapy section). ${ }^{23,24}$ Other agents such as docetaxel and/or irinotecan have demonstrated reasonable clinical activity leading to a modest improvement of survival when compared with best supportive care. ${ }^{25-27}$

In heavily pretreated patients, recent data from the phase 3 TAGS study evaluating the novel nucleoside analogue trifluridine/tipiracil (TAS-102) led to its approval by the Food and Drug Administration (FDA) as third-line 
treatment for advanced EGC. ${ }^{28}$ TAS-102 provided a survival benefit when compared with placebo (mOS 5.7 mos vs 3.6 mos; HR 0.69 [95\% CI $0.56-0.85]$, $\mathrm{p}=0 \cdot 00029$ ), but its effect was largely disease-stabilizing and was associated with a low response rate (ORR $4 \%$ vs $2 \%$ ). Nevertheless, with a manageable toxicity profile, trifluridine/tipiracil provides another option for chemorefractory patients.

\section{Incorporation of Biologics}

Despite the clinical heterogeneity and molecular complexity of EGC, targeted therapeutics directed against multiple mitogenic and angiogenic signaling pathways have been developed and clinically investigated. Clinically relevant targets have included $H E R 2, V E G F(R)$, epidermal growth factor receptor (EGFR), $c M E T$, and more recently the fibroblast growth factor receptor 2 (FGFR2) (Table 1). In this section, we will focus on HER2, FGFR2 and VEGF inhibition.

\section{HER2 Inhibition}

HER2 is a proto-oncogene and member of the ErbB family of receptors that plays a key role in regulation of cellular functions including proliferation, growth, survival, adhesion, migration, and differentiation. HER 2 acts by heterodimerization with other ErbB family receptors leading to activation of the RAS-MAPK (mitogenactivated protein kinase) and PI3K-AKT (phosphatidylinositol 3-kinase-AKT) pathways. In the largest available cohorts using modern assays, HER2 is over-expressed in $\sim 10-20 \%$ of EGC depending on the tumor subtype and location, ${ }^{29}$ with higher incidence in GEJ cancers (GEJC) compared with GC $(32 \%$ vs $21 \%),{ }^{30}$ as well as EAC when compared with ESCC (15-30\% vs 3\%). ${ }^{16}$

Several HER2-targeting agents have been investigated in patients with advanced HER2-positive EGC (Table 1). However, only trastuzumab and trastuzumab deruxtecan (fam-trastuzumab deruxtecan-nxki) have received approval after demonstrating superior efficacy in direct comparisons with standard care.

Trastuzumab is a humanized IgG1 monoclonal antibody $(\mathrm{mAb})$ directed against the extracellular domain of $H E R 2$, and it prevents dimerization of the HER2 receptor. This triggers receptor internalization and mediates antibody-dependent cell-mediated cytotoxicity (ADCC), resulting in inhibition of tumor growth. ${ }^{31}$ In the Phase III ToGA trial, the combination of trastuzumab and cisplatin/ fluoropyrimidine-based chemotherapy (cisplatin/5-FU or cisplatin/capecitabine) was compared with chemotherapy alone as first-line therapy for advanced HER2-positive GEJC/GC. Results of this study indicated an improved overall response rate (ORR; $47 \%$ vs $35 \%$, p $<0.01$ ), progression-free survival (mPFS; 6.7 mos vs 5.5 mos, $\mathrm{p}<0.01$ ), and overall survival (mOS; 13.8 mos vs 11.1 mos; HR $=0.74,95 \%$ CI $0.6-0.91 ; \mathrm{p}<0.01)$ (Table 1$).{ }^{32}$ Based on these results, the FDA approved trastuzumab in 2010, and the combination of trastuzumab with platinum/ fluoropyrimidine-based chemotherapy became the standard of care for frontline treatment of advanced HER2positive GEJC/GC. Several subsequent HER2-targeting agents such as lapatinib, trastuzumab-emtansine (T-DM1), or pertuzumab failed to show a survival benefit. ${ }^{33-36}$ This prompted additional studies evaluating the role of trastuzumab as maintenance therapy (Phase II PLATFORM trial, NCT02678182) or beyond progression on frontline combination therapy. ${ }^{37-39}$ While retrospective studies suggested potential benefit for trastuzumab beyond progression, results of a phase II trial comparing the combination of paclitaxel and trastuzumab vs paclitaxel alone in HER2-positive GEJC/GC refractory to trastuzumabcontaining first-line therapy did not show a survival benefit (mOS 10.2 mos vs 9.95 mos; HR=1.23; 95\% CI $0.75-$ $1.99 ; \mathrm{p}=0.20) .{ }^{40}$ In this study, evaluation of tumor specimen at time of progression on trastuzumab revealed loss of HER2 positivity status in about $2 / 3$ of patients, perhaps contributing to the lack of observed benefit. ${ }^{41}$

Trastuzumab deruxtecan (T-DXd) is an antibody-drug conjugate consisting of an anti-HER2 antibody (trastuzumab), a cleavable tetrapeptide-based linker, and a cytotoxic topoisomerase I inhibitor (deruxtecan). ${ }^{42}$ After internalization, cleavage of the linker by lysosomal enzymes allows topoisomerase I inhibition by deruxtecan, thereby causing DNA damage and apoptosis. Recently, results of the Phase 2 DESTINY-Gastric01 trial evaluating the efficacy of this novel agent compared to physician's choice of either irinotecan or paclitaxel monotherapy in heavily pretreated advanced HER2-positive GEJC/GC showed improved response (ORR $40.5 \%$ vs $11.3 \%$, $\mathrm{p}<0.001$ ) and survival (mOS 12.5 mos vs 8.4 mos; $\mathrm{HR}=0.59 ; 95 \%$ CI $0.39-0.88, \mathrm{p}=0.01$ ) with $\mathrm{T}-\mathrm{DXd}{ }^{43}$ Notable adverse events occurring with T-DXd included myelosuppression and interstitial lung disease (10\%). Based on these data, the US FDA approved T-DXd on January 15, 2021 for patients with advanced HER2positive GEJC/GC who have received a prior trastuzumabbased regimen. While more work is needed to understand 
Table I Summary of Selected Non-Immunotherapy Trials of Antibodies Targeting Key Signaling Pathways in Advanced/Metastatic Esophagogastric Cancer

\begin{tabular}{|c|c|c|c|c|c|c|c|c|c|c|}
\hline Target & $\begin{array}{l}\text { Setting } \\
\text { (Line) }\end{array}$ & Antibody & Study & $\begin{array}{l}\text { Molecular } \\
\text { Selection }\end{array}$ & Intervention & $\mathbf{N}$ & $\begin{array}{l}\text { ORR } \\
{[\%]}\end{array}$ & $\begin{array}{l}\text { PFS } \\
\text { (mos) }\end{array}$ & $\begin{array}{l}\text { OS } \\
\text { (mos) }\end{array}$ & $\begin{array}{l}\text { Met } \\
\text { Primary } \\
\text { Endpoint? }\end{array}$ \\
\hline \multirow[t]{12}{*}{ HER2 } & \multirow[t]{6}{*}{ IL } & \multirow[t]{2}{*}{ Trastuzumab } & ToGA $^{32}$ & \multirow[t]{12}{*}{ Target } & \multirow[t]{2}{*}{$\begin{array}{l}5-\mathrm{FU} / \mathrm{Cis} \pm \\
\text { Trastuzumab }\end{array}$} & \multirow[t]{2}{*}{594} & \multirow[t]{2}{*}{47 vs $35^{b}$} & 6.7 vs 5.5 & $\begin{array}{l}13.8 \mathrm{vs} \\
11.1\end{array}$ & \multirow[t]{2}{*}{ Yes } \\
\hline & & & Phase III & & & & & HR $0.7 I^{b}$ & HR $0.74^{\mathrm{b}}$ & \\
\hline & & \multirow[t]{2}{*}{ Lapatinib } & LOGiC $^{36}$ & & \multirow{2}{*}{$\begin{array}{l}\text { CapeOx + } \\
\text { Lapatinib vs } \\
\text { Placebo }\end{array}$} & \multirow[t]{2}{*}{545} & \multirow[t]{2}{*}{53 vs $39^{b}$} & 6.0 vs 5.4 & $\begin{array}{l}12.2 \text { vs } \\
10.5\end{array}$ & \multirow[t]{2}{*}{ No } \\
\hline & & & Phase III & & & & & $\operatorname{HR} 0.82^{\mathrm{a}}$ & HR 0.9I & \\
\hline & & \multirow[t]{2}{*}{ Pertuzumab } & $\mathrm{JACOB}^{35}$ & & \multirow{2}{*}{$\begin{array}{l}\text { 5-FU/Cis/ } \\
\text { Trastuzumab } \pm \\
\text { Pertuzumab }\end{array}$} & \multirow[t]{2}{*}{780} & \multirow[t]{2}{*}{$\begin{array}{l}56.7 \text { vs } \\
48.3^{\mathrm{a}}\end{array}$} & 8.5 vs 7.0 & $\begin{array}{l}17.5 \text { vs } \\
14.2\end{array}$ & \multirow[t]{2}{*}{ No } \\
\hline & & & Phase III & & & & & HR 0.73 & HR 0.84 & \\
\hline & \multirow[t]{6}{*}{$\geq 2 \mathrm{~L}$} & \multirow[t]{2}{*}{ T-DMI } & GATSBY ${ }^{34}$ & & \multirow[t]{2}{*}{ T-DMI vs Taxane } & \multirow[t]{2}{*}{412} & \multirow[t]{2}{*}{42 vs 20} & 2.7 vs 2.9 & 7.9 vs 8.6 & \multirow[t]{2}{*}{ No } \\
\hline & & & Phase II/ III & & & & & HR I.I3 & HR I.I5 & \\
\hline & & \multirow[t]{2}{*}{$\begin{array}{l}\text { Trastuzumab } \\
\text { deruxtecan }\end{array}$} & $\begin{array}{l}\text { DESTINY- } \\
\text { Gastric0I }\end{array}$ & & \multirow{2}{*}{$\begin{array}{l}\text { Paclitaxel or } \\
\text { Irinotecan } \pm \\
\text { Trastuzumab } \\
\text { deruxtecan }\end{array}$} & \multirow[t]{2}{*}{125} & \multirow[t]{2}{*}{$\begin{array}{l}40.5 \text { vs } \\
11.3^{\mathrm{b}}\end{array}$} & 5.6 vs 3.5 & 12.5 vs 8.4 & \multirow[t]{2}{*}{ Yes } \\
\hline & & & Phase II & & & & & HR 0.47 & $\operatorname{HR} 0.59^{\mathrm{a}}$ & \\
\hline & & \multirow[t]{2}{*}{ Lapatinib } & TyTAN $^{33}$ & & Paclitaxel \pm & 261 & 27 vs $9^{b}$ & 5.5 vs 4.4 & 11.0 vs 8.9 & No \\
\hline & & & Phase III & & & & & HR 0.85 & HR 0.88 & \\
\hline EGFR & IL & Cetuximab & EXPAND $^{126}$ & Unselected & Capecitabine/ & 904 & 30 vs 29 & 4.4 vs 5.9 & 9.4 vs 10.7 & No \\
\hline & & & Phase III & & $\begin{array}{l}\text { Cisplatin } \pm \\
\text { Cetuximab }\end{array}$ & & & HR 1.09 & HR 1.0 & \\
\hline & & Panitumumab & REAL-3 $3^{127}$ & & EOX vs mEOX + & 553 & 46 vs 42 & 6.0 vs 7.4 & 8.8 vs 11.3 & No \\
\hline & & & Phase III & & Panitumumab & & & HR 1.22 & $\operatorname{HR} \quad 1.37^{\mathrm{a}}$ & \\
\hline VEGF-A & IL & Bevacizumab & AVAGAST $^{55}$ & Unselected & $\begin{array}{l}\text { Capecitabine/ } \\
\text { Cisplatin } \pm\end{array}$ & 774 & $\begin{array}{l}46 \text { vs } \\
37.4^{\mathrm{a}}\end{array}$ & 6.7 vs 5.3 & $\begin{array}{l}12.1 \text { vs } \\
10.1\end{array}$ & No \\
\hline & & & Phase III & & Bevacizumab & & & HR $0.80^{\mathrm{b}}$ & HR 0.87 & \\
\hline VEGF- & $2 \mathrm{~L}$ & Ramucirumab & REGARD $^{23}$ & & BSC \pm & 355 & 3.4 vs 2.6 & 2.1 vs 1.3 & 5.2 vs 3.8 & Yes \\
\hline$R 2$ & & & Phase III & & Ramucirumab & & & HR $0.48^{b}$ & HR $0.776^{a}$ & \\
\hline & & & RAINBOW $^{24}$ & & Paclitaxel \pm & 665 & 28 vs $16^{b}$ & 4.4 vs 2.9 & 9.6 vs 7.4 & Yes \\
\hline & & & Phase III & & Ramucirumab & & & HR $0.64^{b}$ & $\operatorname{HR} 0.8 \mathrm{I}^{\mathrm{a}}$ & \\
\hline & & & RAMIRIS $^{56}$ & & FOLFIRI + & 101 & 22 vs II & 4.6 vs 3.6 & 6.8 vs 7.6 & Yes \\
\hline & & & Phase II & & $\begin{array}{l}\text { Ramucirumab vs } \\
\text { Ramucirumab + } \\
\text { Paclitaxel }\end{array}$ & & & HR 0.49 & HR 0.94 & \\
\hline FGFR $2 b$ & IL & Bemarituzumab & $\mathrm{FIGHT}^{50}$ & Target & $\begin{array}{l}\text { mFOLFOX6 } \pm \\
\text { Bemarituzumab }\end{array}$ & 155 & 47 vs 33 & 9.5 vs 7.4 & $\begin{array}{l}\text { NR vs } \\
12.9\end{array}$ & Yes \\
\hline & & & Phase II & & & & & HR 0.68 & HR $0.58^{a}$ & \\
\hline
\end{tabular}

(Continued) 
Table I (Continued).

\begin{tabular}{|c|c|c|c|c|c|c|c|c|c|c|}
\hline Target & $\begin{array}{l}\text { Setting } \\
\text { (Line) }\end{array}$ & Antibody & Study & $\begin{array}{l}\text { Molecular } \\
\text { Selection }\end{array}$ & Intervention & $\mathbf{N}$ & $\begin{array}{l}\text { ORR } \\
{[\%]}\end{array}$ & $\begin{array}{l}\text { PFS } \\
\text { (mos) }\end{array}$ & $\begin{array}{l}\text { OS } \\
\text { (mos) }\end{array}$ & $\begin{array}{l}\text { Met } \\
\text { Primary } \\
\text { Endpoint? }\end{array}$ \\
\hline \multirow[t]{4}{*}{ CMET } & \multirow[t]{4}{*}{ IL } & \multirow[t]{2}{*}{ Onartuzumab } & MetGastric $^{128}$ & \multirow[t]{4}{*}{ Target } & \multirow{2}{*}{$\begin{array}{l}\text { mFOLFOX6 } \pm \\
\text { Onartuzumab }\end{array}$} & \multirow[t]{2}{*}{123} & \multirow{2}{*}{$\begin{array}{l}53.8 \text { vs } \\
44.6\end{array}$} & 6.7 vs 6.8 & 11.0 vs 9.7 & \multirow[t]{2}{*}{ No } \\
\hline & & & Phase III & & & & & HR 0.79 & HR 0.64 & \\
\hline & & \multirow[t]{2}{*}{ Rilotumumab } & RILOMET-I $^{129}$ & & \multirow{2}{*}{$\begin{array}{l}\text { Epirubicin/Cis/ } \\
\text { Cape } \pm \\
\text { Rilotumumab }\end{array}$} & \multirow[t]{2}{*}{450} & \multirow[t]{2}{*}{30 vs 45} & 5.6 vs 6.0 & 8.8 vs 10.7 & \multirow[t]{2}{*}{ No } \\
\hline & & & Phase III & & & & & HR $1.26^{\mathrm{a}}$ & HR $1.34^{\mathrm{b}}$ & \\
\hline
\end{tabular}

Notes: ${ }^{\mathrm{a}} \mathrm{P}<0.05 ;{ }^{\mathrm{b}} \mathrm{P}<0.01$.

Abbreviations: AC, adenocarcinoma; Cis, cisplatin; cape, Capecitabine; EGFR, epidermal growth factor receptor; FGFR2b, fibroblast growth factor receptor 2b; G, gastric; GC, gastric carcinoma; GEJ, gastroesophageal junction; HER2, human epidermal growth factor receptor 2; HR, hazard ratio; mos, months; (m) EOX, (modified) epirubicin, oxaliplatin, Xeloda; mFOLFOX, modified 5-fluorouracil, leucovorin, oxaliplatin; N, number of patients; NR, not reached; ORR, overall response rate; OS, overall survival; Ox, oxaliplatin; PFS, progression-free survival; T-DMI, trastuzumab emtansine; VEGF(R), vascular endothelial growth factor (receptor); IL, Ist line; 2L, 2nd line.

why T-DXd succeeded where the other antibody-drug conjugate T-DM1 failed, putative reasons include the more efficient linker and payload system of T-DXd. Additionally, the high drug-to-antibody ratio of T-DXd and the membrane permeability of its payload make it less dependent on uniformly high HER2 expression, which may be particularly beneficial in the treatment of tumors with lower or heterogeneous HER2 expression. ${ }^{44}$

\section{FGFR2 Inhibition}

The fibroblast growth factor receptors are a family of transmembrane receptors involved in multiple biological processes, including cell proliferation, differentiation, survival, and angiogenesis. FGFR2 amplification has been identified as a poor prognostic factor in approximately 5$10 \%$ of EGC, ${ }^{15,16,45}$ with its FGFR2b splice variant being overexpressed in 3-61\% of EGC depending on tumor stage and assay. ${ }^{46-49}$

Bemarituzumab is a novel humanized $\mathrm{mAb}$ specific to the FGFR $2 b$ receptor that was recently investigated in the phase 2 FIGHT trial with promising results. ${ }^{50}$ This global study enrolled treatment-naive subjects with advanced GC that was not HER2-positive, yet was positive for FGFR2b overexpression or FGFR2 amplification by circulating tumor DNA. Bemarituzumab in combination with mFOLFOX6 led to meaningful improvements in OS (NR vs 12.9 mos; HR $0.58,95 \%$ CI $0.35-0.95, \mathrm{p}=0.0268$ ), PFS (9.5 mos vs 7.4 mos; HR 0.68 , 95\% CI $0.44-1.04$, $\mathrm{p}=0.0727)$, and ORR ( $47 \%$ vs $33 \%)$ when compared with mFOLFOX6 plus placebo. The toxicity profile of bemarituzumab was acceptable, although corneal adverse events and stomatitis were observed in about $30 \%$ of patients. Based on these promising results, further investigation in a global, randomized, double-blind, placebo-controlled Phase 3 study is underway (NCT03343301).

\section{VEGF Pathway Inhibition}

$V E G F$ is a proangiogenic growth factor secreted by the tumor cells in response to hypoxia and nutrient depletion. Upon binding to $V E G F R$, it activates a complex cascade of downstream signaling pathways resulting in neovascularization, vasodilation, and increased vascular permeability. ${ }^{51}$ This aberrant tumor angiogenesis activates immunosuppressive processes that facilitate immune evasion and contribute to tumor survival, migration, and invasion. VEGF and VEGFR have been shown to be overexpressed in $30-40 \%$ of all EGC, ${ }^{52,53}$ and antiangiogenic agents directed against them have demonstrated anti-tumor efficacy alone or in combination with cytotoxic chemotherapy in EGC, with ramucirumab being the only FDA-approved agent to date (Table 1).

Bevacizumab is a recombinant humanized $\mathrm{mAb}$ that acts by specifically binding to the ligand $V E G F-A$, preventing its interaction with $V E G F-R^{54}$ The phase III AVAGAST study, which evaluated bevacizumab in combination with chemotherapy (capecitabine/cisplatin) as firstline therapy in advanced EGC, failed to prolong OS despite significant improvements in median PFS and ORR. $^{55}$

Ramucirumab is a recombinant humanized $\mathrm{mAb}$ that binds to $V E G F-R 2$. Ramucirumab has shown clinical efficacy in randomized phase III studies as a single agent (REGARD) and in combination with paclitaxel (RAINBOW). ${ }^{23,24}$ In REGARD, mOS was modestly improved with ramucirumab compared with placebo (5.2 mos vs 3.8 mos, $p=0.0473$ ) in patients previously treated 
with first-line platinum or fluoro-pyrimidine-based therapy. ${ }^{23}$ A 2.2-month benefit in mOS was observed in RAINBOW with the combination of ramucirumab and paclitaxel compared with paclitaxel alone (9.6 mos vs 7.4 mos, $p=0.0169){ }^{24}$ The results of these studies led to the FDA-approval of ramucirumab either alone or in combination with paclitaxel as second-line treatments for advanced EGC.

Attempts to improve upon the standard of care secondline therapy of ramucirumab alone or in combination with paclitaxel are ongoing. More recently, the phase II RAMIRIS trial evaluated the efficacy of the combination of FOLFIRI and ramucirumab compared with paclitaxel plus ramucirumab for patients with advanced EGC in the second-line setting. One advantage of FOLFIRI/ramucirumab is that it is not neurotoxic, in contrast to paclitaxel/ramucirumab. RAMIRIS failed to show a survival benefit for FOLFIRI/ramucirumab (mOS 6.8 vs 7.6 mos, HR 0.94, $\mathrm{p}=0.77$; mPFS 4.6 vs 3.6 months, HR 0.49, $\mathrm{p}=0.12$ ) in the overall population. However, docetaxel pretreated patients (usually from 1st-line use of FLOT) seemed to derive the most benefit from subsequent FOLFIRI/ramucirumab (mOS 7.5 vs $6.4 \mathrm{mos}, \mathrm{HR}=0.71$, $\mathrm{p}=0.25 ;$ mPFS 4.3 vs $2.0 \operatorname{mos}, \mathrm{HR}=0.72, \mathrm{p}=0.008$ ), prompting an ongoing phase III study to further evaluate this combination (NCT03081143). ${ }^{56}$ Given the comparable survival between paclitaxel/ramucirumab and FOLFIRI/ ramucirumab in RAMIRIS and in another study, ${ }^{57}$ FOLFIRI/ramucirumab has been adopted as a standard option by the National Comprehensive Cancer Network $(\mathrm{NCCN})$.

\section{Immunotherapy}

After almost a decade of impressive clinical efficacy across a wide range of previously untreatable malignancies such as melanoma and lung cancer, clinical activity of immune checkpoint inhibition (ICI) in EGC has emerged in recent years.

Immune checkpoints are negative co-stimulatory proteins expressed on antigen-presenting cells (APC), tumor cells or cytotoxic T-cells. The prototypical targets include the programmed cell death-1 (PD-1) receptor, its ligands PD-L1/L2, and the cytotoxic T-lymphocyte-associated antigen-4 (CTLA-4). Immune checkpoint inhibitors aiming to reverse tumor-associated immunosuppression have been developed and investigated in EGC, with the PD-1/ PD-L1 axis as forerunner target due to PD-L1 expression in approximately $40 \%$ or more of esophagogastric cancers. ${ }^{58}$ PD-L1/L2 are normally expressed on APC and tumor cells, and their binding to PD-1 on activated T cells leads to downregulation of cytotoxic T-cell activity, thereby causing immunosuppression. ${ }^{59}$ Pembrolizumab and nivolumab are humanized mAbs directed against PD-1, which have shown clinical efficacy due to their ability to augment the effector function of tumor-specific CD8+ T-cells, ${ }^{59}$ resulting in tumor rejection. By blocking the interaction between PD-1 and PD-L1/L2, they release PD-1 pathway-mediated inhibition of antitumor immune response, thereby countering immune escape.

\section{3rd Line Treatment}

KEYNOTE-059 and ATTRACTION-2 are landmark studies that led to the first incorporation of ICI in the treatment armamentarium of EGC, including regulatory approvals in September 2017 for pembrolizumab and nivolumab in the US and Japan, respectively (Table 2). The single-arm phase II KEYNOTE-059 trial, which evaluated the efficacy and safety of pembrolizumab monotherapy in patients with advanced GC/GEJC, revealed an ORR of $11.6 \%$ (15.5\% [PD-L1 positive] vs $6.4 \%$ [PD-L1 negative] vs $57.1 \%$ [MSI-H] vs $9 \%$ [non-MSI-H]), mOS of 5.6 mos (5.8 [PD-L1 positive] vs 4.9 [PD-L1 negative]), leading to its approval by the FDA for patients with PD-L1expressing tumors (combined positive score [CPS] $\geq 1$ ) with disease progression on or after two or more systemic therapies. ${ }^{60}$ Similarly, nivolumab was approved in Japan based on the results of the Asian phase 3 ATTRACTION-2 study demonstrating a $37 \%$ reduction in the risk of death $(\mathrm{HR}=0.63 ; 95 \%$ CI $0.51-0.78, \mathrm{p}<0.0001)$ when compared with placebo, in patients with unresectable advanced or recurrent GC/GEJC who had progressed after 2 lines of chemotherapy. ${ }^{61}$ Notably, however, the anti-PD-L1 antibody avelumab did not result in an improvement in OS or PFS compared with chemotherapy in the third-line treatment of GC/GEJC (JAVELIN Gastric 300). ${ }^{62}$

\section{2nd Line Treatment}

The subsequent wave of trials attempting to establish a role for ICI as second-line treatment for metastatic disease has highlighted the heterogeneous biology of these disease entities. Enhanced immunotherapy sensitivity of ESCC, as compared with adenocarcinoma, and the importance of PD-L1 expression in intratumoral immune cells became underscored.

Multiple randomized controlled trials (RCT) showed the activity of PD-1 blockade in PD-L1-unselected ESCC. 


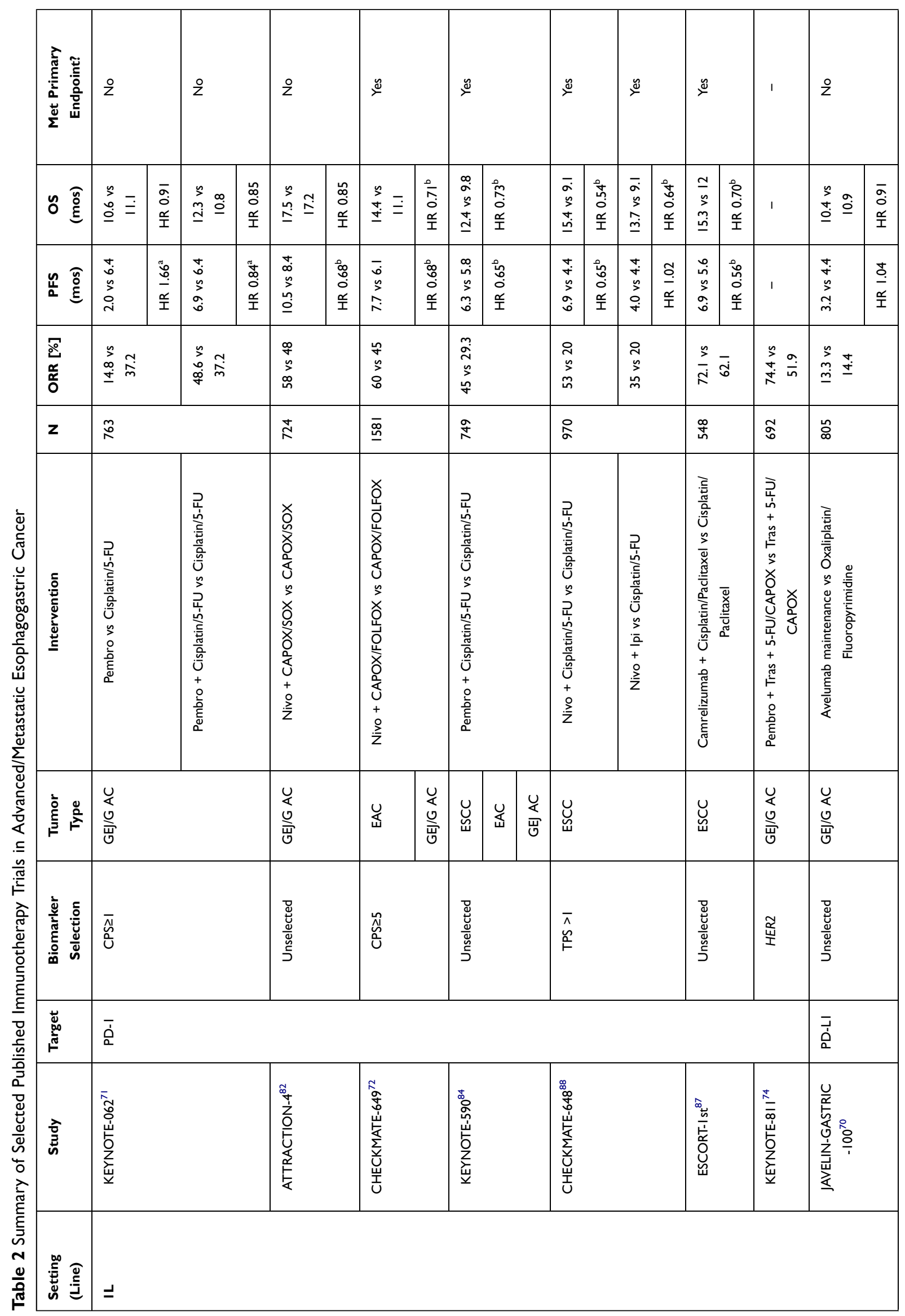




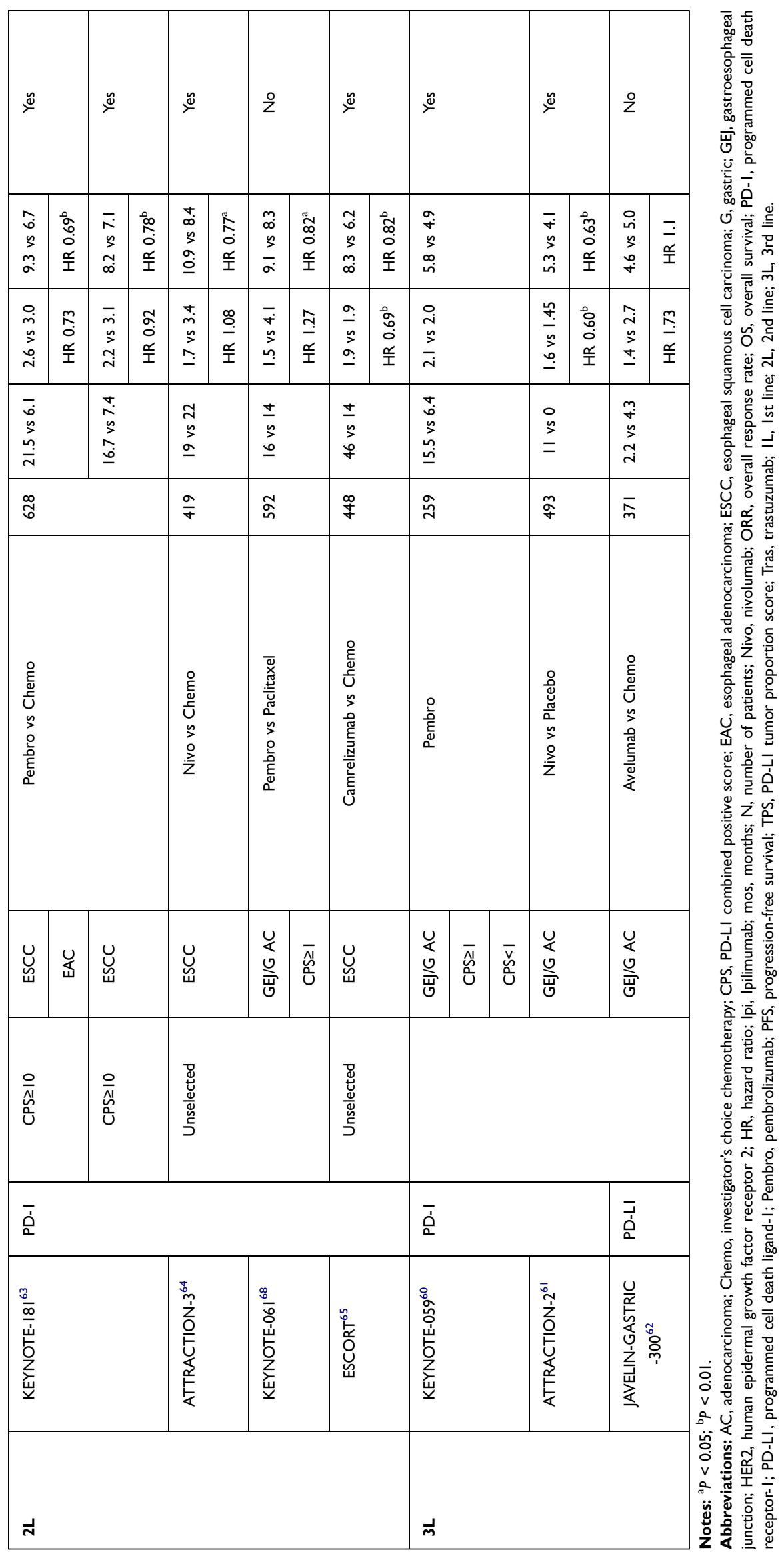


In these studies, which included KEYNOTE-181, ATTRACTION-3, ${ }^{63,64}$ and most recently, ESCORT and RATIONALE-302, ${ }^{65,66}$ PD-1 blockade was directly compared with chemotherapy as second-line treatment in patients with advanced esophageal cancer. The study populations were entirely comprised of squamous cell carcinoma histology, or the positive impact of immunotherapy was most apparent in the ESCC subset.

In KEYNOTE-181, while the co-primary endpoint of OS was not met in the overall population comprising both ESCC and adenocarcinoma and any PD-L1 CPS, the study met its co-primary endpoint of improving OS vs chemotherapy in patients with PD-L1 CPS $\geq 10$ (mOS 9.3 $\operatorname{mos} \quad$ vs $\quad 6.7 \operatorname{mos} \quad[\mathrm{HR}=0.69 ; 95 \% \quad \mathrm{CI}, \quad 0.52-0.93$; $\mathrm{p}=0.0074]$ ). The greatest benefit was observed in ESCC patients with PD-L1 CPS $\geq 10(\operatorname{mOS} 8.2 \mathrm{mos}$ vs $7.1 \mathrm{mos}$ [HR $=0.78 ; 95 \%$ CI, 0.63-0.96; $\mathrm{p}=0.0095])\left(\right.$ Table 2). ${ }^{63}$ This led to the FDA approval in July 2019 of pembrolizumab for patients with advanced PD-L1 CPS $\geq 10$ ESCC with disease progression after one or more prior lines of systemic therapy. In the final subgroup analysis comparing the two histologies, the survival benefit conferred by pembrolizumab over chemotherapy was greater in ESCC compared with EAC, for both patients with PD-L1 CPS $\geq 10$ (HR 0.64 [95\% CI, 0.46-0.9] vs 0.93 [95\% CI, $0.52-$ 1.65]) and in the overall population (HR 0.77 [95\% CI, $0.63-0.96]$ vs 1.12 [95\% CI, 0.85-1.47]). ${ }^{67}$

By contrast, the FDA subsequently approved nivolumab in this indication in June 2020 regardless of PD-L1 expression status, based on findings from the ATTRACTION-3 study. In this Asia-only trial, nivolumab was associated with a $23 \%$ reduction in the risk of death compared with chemotherapy in PD-L1-unselected patients (mOS 10.9 mos vs $8.4 \operatorname{mos}[\mathrm{HR}=0.77 ; 95 \% \mathrm{CI}$ 0.62 to $0.96 ; \mathrm{P}=0.019]$ ) (Table 2$).{ }^{64}$ To our knowledge, outcomes based on PD-L1 CPS have not been reported. Similarly, the ESCORT and RATIONALE studies - also in Asia-only populations - demonstrated a survival benefit of second-line anti-PD-1 antibodies camrelizumab (mOS 8.3 mos vs $6.2 \mathrm{mos}$; HR $=0.71,95 \%$ CI $0.57-0.87)^{65}$ and tislelizumab, ${ }^{66}$ respectively, over investigator's choice of chemotherapy in patients with advanced ESCC. While data from the RATIONALE study has yet to be released, subgroup analysis of the ESCORT study showed that clinical benefits of camrelizumab were observed in all PD-L1 expression subgroups, with the greatest benefit derived in patients with PD-L1 CPS $\geq 5$ (HR for OS 0.51 [95\% CI,
$0.30-0.84]) .{ }^{65}$ In ESCORT, the HR for OS in PD-L1 CPS $<1$ was $0.82(95 \%$ CI $0.62-1 \cdot 09)$ (Table 2). These data further support the activity of PD-1 blockade in patients with advanced ESCC, which is enhanced with higher levels of PD-L1 expression.

By contrast, the global phase 3 KEYNOTE-061 was conducted in an entirely adenocarcinoma population. Pembrolizumab was directly compared with paclitaxel in previously treated patients with PD-L1-positive advanced GC/GEJC (Table 2). Enrollment in the PD-L1 CPS $<1$ group was stopped after a lack of efficacy was determined in this subgroup (mOS 4.8 mos vs 8.2 mos; HR=1.2, 95\% CI $0.89-1.63){ }^{68}$ Then, in the primary efficacy population of PD-L1 CPS $\geq 1$, the trial failed to meet its primary endpoint of OS (mOS $9.1 \mathrm{mos}$ vs $8.3 \mathrm{mos} \mathrm{HR}=0.82,95 \%$ CI $0.66-1.03$; one-sided $\mathrm{p}=0.042){ }^{68}$

A recent small single-arm phase I/II study by Nakajima et al explored the role of concurrent PD-1 and VEGFR2 inhibition as second-line treatment for advanced GC. ${ }^{69}$ It demonstrated manageable toxicities and promising antitumor activity of nivolumab combined with paclitaxel plus ramucirumab with ORR and 6-month PFS rate of 37.2\% (95\% CI, 23.0-53.5\%) and 46.5\% (80\% CI, 36.4\%$55.8 \% ; \mathrm{p}=0.067)$, respectively. Median OS was 13.1 mos (95\% CI, 8.0-16.6 mos) with the most survival benefit observed in patients whose tumor had a PD-L1 CPS $\geq$ 1 (mOS 13.8 mos [95\% CI, 8.0-19.5]), compared to those with PD-L1 CPS $<1$ (mOS 8.0 mos [95\% CI, 4.8-24.1]). With $90.7 \%$ and $32.6 \%$ of patients experiencing grade $\geq 3$ treatment-related and immune-related adverse events, respectively, future studies will be needed to identify the most suitable patients for this combination.

Continued attempts to find a role for ICI in the treatment landscape of advanced EGC led to the investigation of avelumab as a maintenance treatment strategy after front-line therapy in patients with advanced GC/GEJC who had disease control after first-line induction chemotherapy (JAVELIN-GASTRIC-100). ${ }^{70}$ This study did not meet its primary objective of demonstrating superior OS of maintenance avelumab versus continued chemotherapy in the overall or prespecified PD-L1-positive population ( $\geq 1 \%$ of tumor cells). Of note, efficacy results according to PD-L1 expression in immune cells have not been reported to date.

Together, these data from second-line trials comparing anti-PD-1 monotherapy with cytotoxic chemotherapy highlighted the following lessons: 
- Controlling for PD-L1 expression level, ESCCs appear to be more sensitive to PD-1 blockade compared with adenocarcinomas.

- Anti-PD-1 monotherapy results in superior OS compared with chemotherapy in unselected ESCCs, but not in unselected adenocarcinomas.

- Efficacy of anti-PD-1 therapy differs by PD-L1 expression level in immune and tumor cells.

Together, these lessons suggested that future trials in this disease should be interpreted according to histology subtype and PD-L1 immune/tumor expression level.

\section{Ist Line Treatment}

The presence of activity of ICI monotherapy in only a minority of patients indicated that combinatorial approaches might be necessary to enhance ICI efficacy. Given the promising results observed with pembrolizumab and nivolumab monotherapy in later lines of therapy and in selected subgroups, the next generation of trials aimed to test these agents in the first-line metastatic setting in combination with standard chemotherapy regimens and HER2-targeting agents.

The phase III KEYNOTE-062 study failed to demonstrate OS benefit of pembrolizumab with or without chemotherapy vs chemotherapy alone in patients with PD-L1positive (CPS $\geq 1$ ) advanced gastric/gastroesophageal junction cancer (Table 2) ${ }^{71}$ In this global phase 3 study, 763 patients with untreated HER2-negative, PD-L1positive advanced GC/GEJC were randomized in a 1:1:1 ratio to either pembrolizumab alone, pembrolizumab plus cisplatin/fluoropyrimidine chemotherapy, or chemotherapy alone. The study tested multiple primary hypotheses in parallel-ie, superiority of pembrolizumab with chemotherapy versus chemotherapy for PFS and OS in patients with PD-L1 CPS $\geq 1$; superiority of pembrolizumab-chemotherapy for OS in patients with PD-L1 CPS $\geq 10$; and non-inferiority of pembrolizumab versus chemotherapy for OS in patients with PD-L1 CPS $\geq 1$. First, chemotherapy plus pembrolizumab was not superior to chemotherapy alone in tumors with PD-L1 CPS $\geq 1$ (mOS 12.5 mos vs 11.1 mos; HR $=0.85 ; 95 \%$ CI 0.70 1.03; $\mathrm{p}=0.05)$ or $\mathrm{CPS} \geq 10$ (mOS $12.3 \mathrm{mos}$ vs $10.8 \mathrm{mos}$; $\mathrm{HR}=0.85 ; 95 \%$ CI $0.62-1.17 ; \mathrm{p}=0.16$ ). PFS was also not improved from the addition of pembrolizumab to chemotherapy in patients with CPS $\geq 1$ tumors (mPFS 6.9 mos vs 6.4 mos). Second, pembrolizumab monotherapy met the prespecified statistical threshold of non-inferior
OS compared with chemotherapy (mOS 10.6 mos vs 11.1 mos; HR $=0.91,99.2 \%$ CI $0.69-1.18$ ). However, the OS curves were atypical in that the pembrolizumabonly arm initially underperformed chemotherapy, before appearing to outperform chemotherapy later when followup was less mature. Moreover, mPFS was substantially shorter for pembrolizumab than for chemotherapy. Together, these data indicated that the addition of pembrolizumab to front-line cisplatin/FP did not improve OS in PD-L1 CPS $\geq 1$ tumors. Although non-inferiority of pembrolizumab vs chemotherapy was met statistically, questions remain as to whether immunotherapy is truly noninferior. Interestingly, among patients whose tumors had PD-L1 CPS $\geq 10$, the proportion of patients alive at 12 months $(56.5 \%)$ was higher in the pembrolizumab alone group than in the pembrolizumab plus chemotherapy or chemotherapy group (50.5\% and $46.7 \%$, respectively).

In a similar fashion, the global phase III CHECKMATE-649 trial evaluated nivolumab plus chemotherapy (CAPOX/FOLFOX) compared to chemotherapy alone as first-line treatment (Table 2). Key differences in the design from KN-062 were that CHECKMATE-649 had a much larger study population, utilized oxaliplatinbased chemotherapy, did not have an immunotherapy-only arm, and the definition of PD-L1 positivity used a higher cutpoint (CPS $\geq 5$, not CPS $\geq 1$ ). CHECKMATE-649 met both of its co-primary endpoints of OS (14.4 vs $11.1 \mathrm{mos}$; $\mathrm{HR}=0.71,98.4 \%$ CI $0.59-0.86 ; \mathrm{p}<0.0001)$ and PFS (7.7 vs $6.1 \mathrm{mos}$; HR $=0.68,98 \%$ CI $0.56-0.81 ; \mathrm{p}<0.0001)$ in the primary efficacy population of patients expressing PD-L1 CPS $\geq 5$ ( $n=955) .{ }^{72}$ The OS benefit was also statistically significant for the PD-L1 CPS $\geq 1$ population (HR $=0.77$; $\mathrm{p}=0.0001 ; \mathrm{n}=1296)$ and for all randomly assigned patients $(\mathrm{HR}=0.80 ; \mathrm{p}=0.0002 ; \mathrm{n}=1581)$, but the majority of patients in these larger populations had tumors with CPS $\geq 5$. Subgroup analyses suggested a lack of meaningful benefit in patients with tumor PD-L1 CPS 0 ( $\mathrm{n}=265$; mOS: 13.1 mos vs 12.5 mos, HR 0.85 [0.70-1.23]) and CPS <5 ( $\mathrm{n}=$ 606; mOS: 12.4 mos vs 12.3 mos, HR 0.94 [0.78-1.13]). Interaction analysis of OS by PD-L1 CPS cutoffs showed a significant interaction by PD-L1 CPS at the cutoff value of CPS 5 ( $\mathrm{p}=0.011$ ), but not at the cutoff value of CPS of $1 .^{72}$ Nivolumab was recently approved by the FDA in combination with platinum/FP chemotherapy for frontline treatment of patients with advanced or metastatic gastric, gastroesophageal junction or esophageal adenocarcinoma, regardless of PD-L1 status. ${ }^{73}$ However, in light of multiple prior trials in stage IV EGC showing a lack of meaningful 
benefit from immunotherapy in patients with PD-L1-low tumors, ${ }^{68,71,74} \mathrm{NCCN}$ assigned category 1 approval for nivolumab in this setting only in patients whose tumors are CPS $\geq 5$. NCCN assigned Category 2B approval (reflecting a panel vote of $50-84 \%)^{75}$ in patients whose tumors have a PD-L1 CPS $1-4$ and no approval for patients whose tumors have a PD-L1 CPS $<1$.

Why did CHECKMATE-649 succeed where KEYNOTE-062 failed? One speculation to consider is that CHECKMATE-649 primarily tested patients whose tumors had a higher level of PD-L1 expression (CPS $\geq 5$ vs $\geq 1$ in KEYNOTE-062). However, the HR for OS was similarly modest in the KEYNOTE-062 study at the higher cutpoint of CPS $\geq 10$ (ie, HR 0.85). In addition, comparing the CHECKMATE-649 population of CPS $\geq 5$ and the KEYNOTE-062 population at the higher cutpoint of CPS $\geq 10$, none of the efficacy endpoints seemed to be higher in the KEYNOTE-062 study despite using a higher PD-L1 expression cutpoint: HR for PFS was 0.68 in CHECKMATE-649 vs HR 0.73 in KEYNOTE-062. Similarly, the improvement in the median duration of response (DOR) was slightly higher in CHECKMATE649 in patients with CPS $\geq 5$ (9.5 mos with nivolumab vs 7 mos in control), as compared with that in KEYNOTE062 in patients with CPS $\geq 10$ (8.3 mos with pembrolizumab vs 6.8 mos in control). That said, the larger sample size of CHECKMATE-649 provided greater statistical power to analyze subgroups with CPS cutpoints higher than 1. This suggests that the difference in PD-L1 cutpoint may not fully explain the difference in outcomes, unless there is a non-linear association between PD-L1 expression levels and response to immunotherapy.

In addition, patients with MSI-high tumors, which are known to be particularly sensitive to immunotherapy, were more common in KEYNOTE-062 vs CHECKMATE-649 (7.1\% [36/507] among CPS $\geq 1$ in KEYNOTE-062 vs $3.6 \%$ [34/955] among CPS $\geq 5$ in CHECKMATE), suggesting a higher frequency of MSI-H would not explain the discrepancy.

Other characteristics were comparable between the two study populations: proportion of patients enrolled from Asia ( $\sim 25 \%$ each), median age ( $\sim$ age $62-63)$, proportion of patients with ECOG PS 1 vs $0(\sim 54-59 \%)$ or with gastric tumor location vs GEJ or esophagus $(\sim 66-72 \%$ each). The proportion of patients with high tumor mutational burden (TMB) has not yet been reported.

Another possibility is the choice of platinum chemotherapy (oxaliplatin vs cisplatin). Oxaliplatin is known to induce immunogenic cell death in experimental systems, ${ }^{76}$ whereas some studies ${ }^{77-79}$ - but not all ${ }^{81}$ suggest cisplatin may be suboptimal in its ability to induce immunogenic cell death due in part to its failure to induce calreticulin exposure on the cell surface.

Finally, the proportion of patients in the control arm that received subsequent immunotherapy was higher in KEYNOTE-062 (13.5\%) as compared with CHECKMATE (8\%), potentially diluting the observed OS benefit in KEYNOTE. However, whether subsequent immunotherapy exposure after front-line therapy provides benefit is unestablished. This is particularly the case, given the results of KN061 showing a lack of benefit from second-line immunotherapy vs second-line chemotherapy (after front-line chemotherapy) and of JAVELIN Gastric 100 showing a lack of benefit from maintenance immunotherapy vs chemotherapy after disease stability on front-line chemotherapy.

In contrast to CHECKMATE-649, the phase III ATTRACTION-4 study failed to show OS benefit with the addition of nivolumab to frontline chemotherapy. This study randomized an entirely Asian (Japan, Korea, and Taiwan) cohort of 724 patients with untreated HER2negative advanced gastric/gastroesophageal junction adenocarcinoma to either nivolumab in combination with chemotherapy (oxaliplatin $+\mathrm{S}-1$ [SOX] or capecitabine [CAPOX]) vs placebo in combination with chemotherapy (Table 2). ${ }^{82}$ While the addition of nivolumab to chemotherapy resulted in an improvement in one of the coprimary endpoints of PFS (mPFS 10.45 mos vs 8.35 mos; HR $0.68,98.51 \%$ CI $0.51-0.90 ; \mathrm{p}=0.0007$ ) and elicited higher ORR (57.5\% vs $47.8 \%$ ), there was no statistically significant improvement in the other co-primary endpoint of OS (mOS 17.45 mos vs 17.15 mos; HR 0.90, 95\% CI 0.75-1.08; $\mathrm{p}=0.257$ ).

Potential explanations for why CHECKMATE-649 showed an OS benefit but ATTRACTION-4 did not include the following: ATTRACTION-4 had a smaller sample size (1581 vs 724 patients) and enrolled from a different geographic region (global vs Asian). Patients treated in Asia are known to be more likely to receive multiple lines of therapy, as compared with non-Asia regions, which may have diluted the impact of front-line therapy. In particular, $27 \%$ of patients in the control arm of ATTRACTION-4 received post-study immunotherapy, vs only $8 \%$ of patients in the control arm of CHECKMATE649. Furthermore, whether one study population had more vs fewer immunogenic tumors cannot be accurately determined, as methods of biomarker assessment used in the 2 
trials differed. The primary endpoint in CHECKMATE649 was assessed based on PD-L1 CPS (which accounts for PD-L1 on tumor and tumor associated immune cells), whereas biomarker assessment in ATTRACTION-4 was made based on PD-L1 tumor proportion score (TPS). TPS has been a less reliable predictor for immunotherapy benefit than CPS in EGC. ${ }^{83}$

The first large phase III trial testing PD-1 blockade in previously untreated patients with advanced ESCC was KEYNOTE-590. This global study examined pembrolizumab in combination with chemotherapy vs placebo plus chemotherapy in the frontline treatment of patients with advanced esophageal cancer, mostly comprising ESCC but also including adenocarcinoma of the distal esophagus and GEJ (ie, Siewert 1) (Table 2). ${ }^{84}$ The study met its coprimary endpoints of superiority for PFS and OS of pembrolizumab-chemotherapy in PD-L1 unselected ESCC, and for OS in ESCC CPS $\geq 10$. The benefits were highest for ESCC patients with PD-L1 CPS $\geq 10$ (mOS $13.9 \mathrm{mos}$ vs 8.8 mos; HR $0.57,95 \%$ CI $0.43-0.75$; $p<0.0001)$. In the full unselected ESCC cohort, OS was also improved (mOS 12.6 mos vs $9.8 \mathrm{mos} ; \mathrm{HR}=0.72,95 \%$ CI $0.60-0.88$; $\mathrm{p}<0.0006$ ). In the overall population that included both ESCC and adenocarcinoma, first-line pembrolizumab plus chemotherapy provided a statistically significant and clinically meaningful improvement in OS (12.4 mos vs 9.8 mos; HR $=0.73$, 95\% CI $0.62-0.86 ; \mathrm{p}<0.0001)$ and PFS (6.3 mos vs 5.8 mos; HR $=0.65$, 95\% CI $0.55-0.76$; $\mathrm{p}<0.0001$ ), as well as ORR. Exploratory analysis indicated a lack of benefit in patients with PD-L1 CPS $<10$ (ESCC and adenocarcinoma pooled: mOS 10.5 mos vs $10.6 \mathrm{mos}$; HR $0.86,95 \%$ CI $0.68-1.10) .{ }^{85}$ Detailed outcomes within ESCC or adenocarcinoma with CPS $<10$ have not been reported to date. Based on these data, NCCN assigned category 1 or $2 \mathrm{~A}$ approval (indicating uniform $[\geq 85 \%]$ consensus) for pembrolizumab in combination with platinum/FP chemotherapy in this setting only in patients whose tumors are CPS $\geq 10$ regardless of histology. Category $2 \mathrm{~B}$ approval ${ }^{75}$ was assigned to patients with adenocarcinoma with PD-L1 CPS 1-9, and no approval for patients whose tumors have a PD-L1 CPS $<1$. By contrast, the FDA's Oncology Center of Excellence announced broader approval of pembrolizumab in combination with platinum/FP chemotherapy for patients with metastatic or locally advanced esophageal or GEJ (tumors with epicenter 1-5 centimeters above the GEJ) carcinoma who are not candidates for surgical resection or definitive chemoradiation, regardless of PD-L1 expression. ${ }^{85}$
In light of the approval of ICI in combination with chemotherapy in first-line setting of metastatic or advanced EGC (based on CHECKMATE-649 and KEYNOTE-590), and the failure of confirmatory benefit for single-agent PD-1 inhibition over chemotherapy in the second-line and first-line settings for adenocarcinoma patients (KEYNOTE-061 and KEYNOTE-062, respectively), the FDA's Oncologic Drugs Advisory Committee (ODAC) recently reversed the initial approval of pembrolizumab in the 3rd line setting for patients with tumor PDL1 CPS $\geq 1$. $^{86}$

Further data supporting the OS benefit of adding PD-1 blockade in patients with previously untreated ESCC was observed in the phase 3 ESCORT-1st study. In this study, frontline camrelizumab plus chemotherapy was compared with placebo plus chemotherapy in Chinese patients with advanced or metastatic ESCC. Preliminary results were presented at the 2021 ASCO Annual Meeting. In this study, camrelizumab plus chemotherapy led to an improvement in both co-primary endpoints of OS (15.3 mos vs 12.0 mos; $\mathrm{HR}=0.70,95 \%$ CI $0.56-0.88 ; \mathrm{p}=0.001$ ) and PFS $(6.9$ mos vs 5.6 mos; HR $=0.56$, 95\% CI $0.46-$ 0.68 ; $\mathrm{p}<0.001)$ when compared with placebo plus chemotherapy (Table 2). Subgroup analysis was performed that included stratification by PD-L1 expression level, but it was not clearly reported whether PD-L1 expression was assessed in tumor cells, immune cells, or both. Greatest benefit was observed in patients with PD-L1 $\geq 10 \%$ ( $\mathrm{n}=104$ in camrelizumab and $\mathrm{n}=98$ in placebo; HR 0.52 [95\% CI, 0.35-0.79]), as compared with PD-L1 $<10 \%(\mathrm{n}=188$ and $\mathrm{n}=195$, respectively; HR $0.78[95 \%$ CI, 0.59-1.02]), PD-L1 $<5 \%(\mathrm{n}=145$ and 155 , respectively; HR 0.77 [95\% CI, 0.56-1.04]) or PD-L1 <1\% $(\mathrm{n}=126$ and $\mathrm{n}=130$, respectively; HR 0.79 [95\% CI, $0.57-1.11]) .{ }^{87}$

Lastly, recently presented data from the phase III CHECKMATE-648 study, which evaluated the efficacy of doublet chemotherapy (cisplatin/FP) alone vs in combination with nivolumab or combination with nivolumab and ipilimumab in the first-line setting in 970 patients with unresectable advanced or metastatic ESCC, revealed a statistically significant and clinically meaningful OS benefit with the addition of nivolumab to either chemotherapy or ipilimumab, compared with chemotherapy alone, among patients with tumor PD-L1 TPS $\geq 1 \%$ (15.4 mos vs 9.1 mos $[\mathrm{HR}=0.54, \quad 99.5 \%$ CI $\quad 0.37-0.80$; $\mathrm{p}<0.0001]$ and $13.7 \mathrm{mos}$ vs $9.1 \mathrm{mos}[\mathrm{HR}=0.64,98.6 \% \mathrm{CI}$ $0.46-0.90 ; \mathrm{p}=0.001]$, respectively) and in the all- 
randomized patient population (13.2 mos vs $10.7 \mathrm{mos}$ [HR $=0.74,99.1 \%$ CI $0.58-0.96 ; \mathrm{p}=0.0021]$ and $12.8 \mathrm{mos}$ vs $10.7 \operatorname{mos}$ [HR $=0.78,98.2 \%$ CI $0.62-0.98 ; \mathrm{p}<0.0011$ ], respectively) (Table 2). ${ }^{88}$ This OS benefit was not observed in patients with tumor PD-L1 TPS $<1 \%$ when comparing nivolumab plus chemotherapy (HR 0.98; median OS $12.0 \mathrm{mos}$ vs $12.2 \mathrm{mos}$ ) and nivolumab plus ipilimumab (HR 0.96; median OS 12.0 mos vs 12.2 mos) to chemotherapy alone. While PFS of nivolumab plus ipilimumab vs chemotherapy alone in patients with PD-L1 TPS $\geq 1 \%$ did not meet the prespecified boundary for significance, a statistically significant PFS was observed with the addition of nivolumab to chemotherapy vs chemotherapy alone (HR 0.65 [98.5\% CI 0.46-0.92]; $\mathrm{P}=$ 0.0023 ) in patients with PD-L1 TPS $\geq 1 \%{ }^{88}$ Higher ORR was also observed with nivolumab plus chemotherapy and nivolumab plus ipilimumab, compared with chemotherapy alone, in both patients with PD-L1 TPS $\geq 1 \%$ ( $53 \%$ vs $35 \%$ vs $20 \%$, respectively). ORR in TPS $<1 \%$ has not reported to date. ${ }^{88}$ Although no new safety signals were identified, grade 3-4 toxicity leading to discontinuation was higher for nivolumab-ipilimumab vs chemotherapy ( $13 \%$ vs $5 \%)$, particularly after accounting for the shorter treatment duration of nivolumab-ipilimumab. These data suggest that these chemoimmunotherapy and dual-immunotherapy combinations may be potential firstline options for selected patients with unresectable advanced or metastatic ESCC whose tumors are TPS $>1 \%$. It will be helpful if future analyses include outcomes data based on PD-L1 CPS.

In advanced HER2-positive GEJC/GC, a number firstline doublet and triplet combinations have been investigated or subject of ongoing investigations, notably, in the MAHOGANY and PANTHERA studies. The single-arm phase Ib/II MAHOGANY study (CP-MGAH22-05) evaluated the antitumor activity of margetuximab, an Fcengineered HER2-directed $\mathrm{mAb}$ with enhanced ADCC activity, ${ }^{89-91}$ in combination with PD-1 inhibitor pembrolizumab in the HER2-positive, PD-L1-unselected patients who had progressed after at least one prior line of therapy with trastuzumab plus chemotherapy. ${ }^{92}$ This study revealed an acceptable safety and tolerability profile as well as clinically meaningful outcomes including ORR of $18.48 \%$ (95\% CI, 11.15-27.93), disease control rate (DCR) of $53 \%$ (95\% CI, 43-64\%), mPFS of $2.73 \mathrm{mos}$ (95\% CI, 1.61-4.34), and mOS of $12.48 \operatorname{mos}(95 \% \mathrm{CI}$, 9.07-14.09). Subgroup analysis revealed higher ORR of $44 \%$ (95\% CI, 24-65) and DCR of 72\% (95\% CI, 51-
$88 \%$ ) in the double-positive subgroup (HER2 IHC3positive/PD-L1-positive) comprising 32\% of the study population. This promising activity prompted the design of the ongoing randomized, open-label phase II/III MAHOGANY study (NCT04082364) to further explore the prospect of margetuximab plus retifanlimab (anti-PD-1 IgG4 $\mathrm{mAb}$ ) with/without chemotherapy and margetuximab plus tebotelimab (anti-PD-1 and anti-LAG-3 bispecific IgG4 $\mathrm{mAb}$ ) with chemotherapy in the first-line treatment of GC/GEJ adenocarcinoma. ${ }^{93}$ Additionally, the Korean multicenter, single-arm phase Ib/II PANTHERA trial evaluated the triple combination of pembrolizumab, trastuzumab, and chemotherapy as first-line therapy for advanced HER2-positive GEJC/GC. Data recently reported at the 2021 ASCO GI symposium revealed promising activity in PD-L1-unselected patients with ORR of $76.7 \%(95 \%$ CI, 61.4-88.2), mPFS of 8.6 mos (95\% CI, 7.2-16.5), mOS of 19.3 mos (95\% CI, 16.5-NR), and DOR of 10.8 mos (95\% CI, 7.2-21.8). ${ }^{94}$ Building on this encouraging activity, the ongoing global phase III KEYNOTE-811 trial is investigating the addition of pembrolizumab vs placebo to trastuzumab and chemotherapy in treatment-naive, advanced HER2-positive GEJC/GC (NCT03615326). The first prespecified interim analysis recently presented at the 2021 ASCO Annual Meeting revealed an acceptable safety profile and a statistically significant improvement in ORR with the addition of pembrolizumab to trastuzumab plus chemotherapy when compared with placebo $(74.4 \%$ vs $51.9 \%$; 95\% CI $[11.2,33.7] ; \mathrm{p}=0.00006)($ Table 2$) .{ }^{74}$ While the dual primary endpoints of PFS and OS were not reported and are anticipated in upcoming analyses, these interim results have led to the FDA granting accelerated approval for pembrolizumab in combination with trastuzumab plus chemotherapy as first-line therapy for advanced HER2-positive GEJC/GC.

\section{Future Directions}

Despite recent advances, outcomes in most patients with advanced EGC remain quite poor. This is particularly true for the majority of patients who lack expression of any known targetable anomaly-ie, HER2 negative, PD-L1 negative. In this regard, a recent exome, transcriptome, immune profile analysis identified multiple independent prognostic factors in EGC discriminating longer from shorter survivors. ${ }^{95}$ A shorter survivor subtype was found to be associated with aggressive phenotypes and distinct expression features (eg, upregulated druggable targets $J A K 2, M A P 3 K 13$ and $M E C O M$ ), whereas a long survivor 
subtype exhibited intact $C h r 4$ and high $A P O B E C$ mutation signature with potentially greater benefit from immunotherapy ${ }^{95}$ In addition, recent data provides compelling evidence for targeting Claudin18.2 (CLDN18.2), a gastric-specific tight junction protein exposed during tumorigenesis that may be enriched in HER2-negative tumors. The randomized phase 2 FAST study evaluating the chimeric $\mathrm{mAb}$ Zolbetuximab in combination with firstline EOX in patients with advanced CLDN18.2-positive $\mathrm{GC} / \mathrm{GEJC} / \mathrm{EAC}$ revealed a significant PFS (HR $=0.44,95 \%$ CI $0.29-0.67 ; \mathrm{p}<0.0005)$ and $\mathrm{OS}(\mathrm{HR}=0.55,95 \%$ CI 0.39 $0.77 ; \mathrm{p}<0.0005)$ benefit when compared with EOX alone. $^{96}$ This agent is currently being evaluated in two phase 3 trials (NCT03504397, NCT03653507).

To improve on the recent success of chemoimmunotherapy combinations, it will be important to understand of the mechanisms of therapeutic resistance which can inform more effective anti-cancer therapeutic partners. One strategy to enhance ICI response targeted immunosuppressive macrophages and angiogenesis (LEAP-005 study) through the combination of pembrolizumab plus lenvatinib, an oral multi-kinase inhibitor that has been shown to inhibit tumor-associated macrophages and enhance the activation of the interferon signaling pathway. ${ }^{97}$ While an ORR of $9.7 \%$ was observed in this study, ${ }^{98}$ this combination yielded a much higher ORR of $69 \%$ in Asian phase 2 study, ${ }^{99}$ providing rationale for further investigation in a larger global study. Furthermore, a subset of patients whose tumor express PD-L1 also co-express HER2. Preliminary data from dual targeting of HER2 and PD-L1 have shown promising results, ${ }^{100}$ and this approach is undergoing further evaluation in phase 3 trials (NCT04082364, NCT03615326). Lastly, novel combinatorial approaches incorporating agents targeting other immune checkpoint pathways, such as TIM3 (T-cell immunoglobulin and mucin domain containing-3), LAG3 (Lymphocyte-activation gene 3) and TIGIT (T cell immunoreceptor with Ig and ITIM domains) could help overcome ICI resistance by restoring effector function of exhausted T cells. ${ }^{101}$ Dual checkpoint inhibitor combination therapy with nivolumab and relatlimab (LAG-3-blocking antibody) recently demonstrated benefit in a phase III clinical trial of advanced melanoma, ${ }^{102}$ and the results of trials evaluating this combination in other tumors including GC (NCT02935634) are awaited.

Furthermore, cell-based approaches such as chimeric antigen receptor (CAR) T cell therapy and T-cell receptor (TCR) therapy are therapeutically well suited for clinically heterogeneous and molecularly complex cancers such as EGC, and have the potential to overcome the therapeutic resistance related to the plasticity of the molecular targets. ${ }^{103,104}$ Targets currently being investigated include HER 2, carcinoembryonic antigen (CEA), mucin 1 (MUC1), epithelial cell adhesion molecule (EpCAM), $C L D N$ 18.2, mesothelin $(M S L N)$, natural-killer receptor group 2, member $\mathrm{D}(N K G 2 D)$, and folate receptor 1 (FOLR1). ${ }^{105-113}$ Promising activity was demonstrated with $C L D N 18.2$ in the ongoing first-in-human Phase 1 study evaluating autologous CAR-CLDN18.2 $\mathrm{T}$ cells in patients with advanced gastric and pancreatic adenocarcinoma. This study included 12 patients (5 pancreatic and 7 gastric), and preliminary results revealed that CARCLDN18.2 T cell therapy was safe and well tolerated, and yielded an ORR of 33\% (including 1 complete and 2 partial responses in the GC cohort) and mPFS of 130 days. ${ }^{114}$

It is critical to improve our understanding of the impact of immunotherapy on the tumor microenvironment. In this regard, most randomized trials directly comparing immunotherapy with chemotherapy in EGC have shown a noticeably shorter PFS with immunotherapy, despite more comparable median OS. This phenomenon is likely explained in part by the small subset of patients who experience durable benefit from immunotherapy alone. However, the small subset of extraordinary responders is unlikely to explain the improvement in median OS. The discrepancy between PFS and OS results has raised the possibility that immunotherapy-experienced patients may disproportionately benefit from subsequent therapy. A number of studies by our group and others have suggested that response to subsequent therapy following immunotherapy may be enhanced in association with alterations in the immune microenvironment. ${ }^{115-120}$ However, these hypothesis-generating data require prospective validation, which is underway, in multiple ongoing and planned studies (NCT04069273).

Lastly, because of the clinical heterogeneity and molecular complexity of EGC, future precision medicine efforts should be directed towards the identification of better predictive biomarkers to not only facilitate selection of patients most likely to benefit from the emerging novel targeted and immunotherapy regimens, but also improve patient stratification for clinical trial design. In addition to PD-L1 expression, MSI status has been shown to be a robust tumor-agnostic predictor of response to immunotherapy, with MSI-high tumors demonstrating 
considerable and durable survival benefit. Other markers (eg, DNA damage pathways) that are associated with neoantigen production are currently being investigated. Some data suggest that EBV-positive GCs, which appear to have enhanced immune cell infiltration and PD-L1/2 expression, may be more sensitive to PD-1/PD-L1 inhibition, although this requires further study. ${ }^{15,121-123}$ Evaluation of the determinants of response to immunotherapy in a phase II biomarker trial of salvage pembrolizumab in 61 patients with metastatic GC revealed an impressive ORR in both the MSI-high (85.7\%) and EBVpositive $(100 \%)$ cohorts, although sample sizes were limited. ${ }^{124}$ More recently, a Korean phase II biomarkerintegrated umbrella study evaluating the combination of second-line nivolumab and paclitaxel in 48 patients with EBV-related, dMMR/MSI-high or PD-L1-positive advanced GC revealed an ORR of $25 \%$ in the EBVpositive cohort $(n=5)$, with an ongoing complete response after 24 months of therapy. ${ }^{125}$ Further study will be needed to better understand the prognostic role of EBV-positivity in $\mathrm{GC}$.

\section{Conclusion}

In the last few years, there has been a dramatic expansion of therapies that have improved outcomes in patients with advanced EGC. This includes immunotherapies that provide substantial benefit in a minority of patients (eg, MSI-high), as well as combination therapies that have incrementally improved outcomes in a larger group of patients (eg, PD-L1- or HER2-expressing). The most effective biologic agents, to date, have been those that target HER2, PD-1, and VEGFR2. However, other promising targets have been identified including FGFR2, Claudin18.2, and others. An enormous amount of work remains to be done, as outcomes continue to remain poor. Moving forward, approaches that may have the highest potential for immediate clinical impact may include therapies that target patients whose tumors show coexpression or -alteration of multiple targets (eg, "doublepositive"); identifying novel targets for patients with the most aggressive disease; and further understanding how the immune microenvironment inhibits and/or potentiates the impact of various agents.

\section{Acknowledgments}

This work was supported by CTSA Grant Number UL1 TR002377 from the National Center for Advancing Translational Science (NCATS). Its contents are solely the responsibility of the authors and do not necessarily represent the official views of the NIH.

\section{Disclosure}

HHY reports grant, research, and/or clinical trial support from Merck \& Co., Inc, Macrogenics, Bristol Myer Squibb; and honoraria (advisory boards and/or steering committees) from Merck, Macrogenics, BeiGene, ALX Oncology, Bristol Myer Squibb, AstraZeneca and Advisory/Consultant (all paid to institution) from Astellas, OncXerna, and Zymeworks, outside the submitted work. The authors report no other conflicts of interest in this work.

\section{References}

1. Siegel RL, Miller KD, Jemal A. Cancer statistics, 2019. $C A$ Cancer J Clin. 2019;69(1):7-34. doi:10.3322/caac.21551

2. Torre LA, Siegel RL, Ward EM, et al. Global cancer incidence and mortality rates and trends-an update. Cancer Epidemiol Biomarkers Prev. 2016;25(1):16-27. doi:10.1158/1055-9965. EPI-15-0578.

3. Noone AM, Howlader N, Krapcho M, et al. SEER cancer statistics review, 1975-2015; 2018, National Cancer Institute. Bethesda, MD. based on November 2017 SEER data submission, posted to the SEER web site, April 2018. Available from: https:// seer.cancer.gov/csr/1975_2015/. Accessed July 14, 2021.

4. Rustgi AK, El-Serag HB, Ingelfinger JR. Esophageal carcinoma. N Engl J Med. 2014;371:2499-2509. doi:10.1056/NEJMra1314530

5. Freedman ND, Abnet CC, Caporaso NE, et al. Impact of changing US cigarette smoking patterns on incident cancer: risks of 20 smoking-related cancers among the women and men of the NIHAARP cohort. Int $J$ Epidemiol. 2016;45(3):846-856. doi:10.1093/ije/dyv175

6. Islami F, Fedirko V, Tramacere I, et al. Alcohol drinking and esophageal squamous cell carcinoma with focus on light-drinkers and never-smokers: a systematic review and meta-analysis. Int $J$ Cancer. 2011;129(10):2473-2484. doi:10.1002/ijc.25885

7. Lauby-Secretan B, Scoccianti C, Loomis D, et al. Body fatness and cancer-viewpoint of the IARC working group. $N$ Engl $J$ Med. 2016;375(8):794-798. doi:10.1056/NEJMsr1606602

8. Thrift AP, Shaheen NJ, Gammon MD, et al. Obesity and risk of esophageal adenocarcinoma and Barrett's esophagus: a Mendelian randomization study. J Natl Cancer Inst. 2014;106 (11):dju252. doi:10.1093/jnci/dju252

9. Peleteiro B, Bastos A, Ferro A, et al. Prevalence of Helicobacter pylori infection worldwide: a systematic review of studies with national coverage. Dig Dis Sci. 2014;59(8):1698-1709. doi:10.1007/s10620-014-3063-0

10. Ford AC, Forman D, Hunt RH, et al. Helicobacter pylori eradication therapy to prevent gastric cancer in healthy asymptomatic infected individuals: systematic review and meta-analysis of randomised controlled trials. BMJ. 2014;348:g3174. doi:10.1136/ bmj.g3174

11. Doorakkers E, Lagergren J, Engstrand L, et al. Helicobacter pylori eradication treatment and the risk of gastric adenocarcinoma in a Western population. Gut. 2018;67(12):2092-2096. doi:10.1136/gutjnl-2017-315363

12. Chey WD, Leontiadis GI, Howden CW, et al. ACG clinical guideline: treatment of Helicobacter pylori infection. $\mathrm{Am}$ J Gastroenterol. 2017;112(2):212-239. doi:10.1038/ajg.2016.563 
13. Liyanage SS, Rahman B, Ridda I, et al. The aetiological role of human papillomavirus in oesophageal squamous cell carcinoma: a meta-analysis. PLoS One. 2013;8(7):e69238. doi:10.1371/journal. pone. 0069238

14. Rajendra S, Xuan W, Merrett N, et al. Survival rates for patients with Barrett high-grade dysplasia and esophageal adenocarcinoma with or without human papillomavirus infection. JAMA Netw Open. 2018;1(4):e181054. doi:10.1001/jamanetworkopen.2018.1054

15. The Cancer Genome Atlas Research Network. Comprehensive molecular characterization of gastric adenocarcinoma. Nature. 2014;513:202-209. doi:10.1038/nature13480

16. Cancer Genome Atlas Research Network. Integrated genomic characterization of oesophageal carcinoma. Nature. 2017;541 (7636):169. doi:10.1038/nature20805

17. Janmaat VT, Steyerberg EW, van der Gaast A, et al. Palliative chemotherapy and targeted therapies for esophageal and gastroesophageal junction cancer. Cochrane Database Syst Rev. 2017;11:Cd004063. doi:10.1002/14651858.CD004063.pub4

18. Al-Batran S, Hartmann J, Probst S, et al. Phase III trial in metastatic gastroesophageal adenocarcinoma with fluorouracil, leucovorin plus either oxaliplatin or cisplatin: a study of the Arbeitsgemeinschaft Internistische Onkologie. J Clin Oncol. 2008;26:1435-1442. doi:10.1200/JCO.2007.13.9378

19. Cunningham D, Starling N, Rao S, et al. Capecitabine and oxaliplatin for advanced esophagogastric cancer. $N$ Engl $J$ Med. 2008;358:36-46. doi:10.1056/NEJMoa073149

20. Bang YJ, Van CE, Feyereislova A, et al. Trastuzumab in combination with chemotherapy versus chemotherapy alone for treatment of HER2-positive advanced gastric or gastro-oesophageal junction cancer (ToGA): a phase 3, open-label, randomised controlled trial. Lancet. 2010;376:1302. doi:10.1016/S0140-6736(10)61121-X

21. Guimbaud R, Louvet C, Ries P, et al. Prospective, randomized, multicenter, phase III study of fluorouracil, leucovorin, and irinotecan versus epirubicin, cisplatin, and capecitabine in advanced gastric adenocarcinoma: a French intergroup (Federation Francophone de Cancerologie Digestive, Federation Nationale des Centres de Lutte Contre le Cancer, and Groupe Cooperateur Multidisciplinaire en Oncologie) study. $J$ Clin Oncol. 2014;32:3520-3526. doi:10.1200/jco.2013.54.1011

22. Dank M, Zaluski J, Barone C, et al. Randomized phase III study comparing irinotecan combined with 5-fluorouracil and folinic acid to cisplatin combined with 5-fluorouracil in chemotherapy naive patients with advanced adenocarcinoma of the stomach or esophagogastric junction. Ann Oncol. 2008;19:1450-1457. doi:10.1093/annonc/mdn 166

23. Fuchs CS, Tomasek T, Yong CJ, et al. Ramucirumab monotherapy for previously treated advanced gastric or gastro-oesophageal junction adenocarcinoma (REGARD): an international, randomised, multicentre, placebo-controlled, phase 3 trial. Lancet. 2014;383:31-39. doi:10.1016/s0140-6736(13)61719-5

24. Wilke H, Muro K, Van Cutsem E, et al. Ramucirumab plus paclitaxel versus placebo plus paclitaxel in patients with previously treated advanced gastric or gastro-oesophageal junction adenocarcinoma (RAINBOW): a double-blind, randomised phase 3 trial. Lancet Oncol. 2014;15:1224-1235. doi:10.1016/s1470-2045(14)70420-6

25. Ford HE, Marshall A, Bridgewater JA, et al. Docetaxel versus active symptom control for refractory oesophagogastric adenocarcinoma (COUGAR-02): an open-label, phase 3 randomised controlled trial. Lancet Oncol. 2014;15:78-86. doi:10.1016/s14702045(13)70549-7

26. Thuss-Patience PC, Kretzschmar A, Bichev D, et al. Survival advantage for irinotecan versus best supportive care as secondline chemotherapy in gastric cancer-a randomised phase III study of the Arbeitsgemeinschaft Internistische Onkologie (AIO). Eur J Cancer. 2011;47:2306-2314. doi:10.1016/j.ejca.2011.06.002
27. Hironaka S, Ueda S, Yasui $\mathrm{H}$, et al. Randomized, open-label, phase III study comparing irinotecan with paclitaxel in patients with advanced gastric cancer without severe peritoneal metastasis after failure of prior combination chemotherapy using fluoropyrimidine plus platinum: WJOG 4007 trial. J Clin Oncol. 2013;31:4438-4444. doi:10.1200/jco.2012.48.5805

28. Shitara K, Doi T, Dvorkin M, et al. Trifluridine/tipiracil versus placebo in patients with heavily pretreated metastatic gastric cancer (TAGS): a randomised, double-blind, placebo-controlled, phase 3 trial. Lancet Oncol. 2018;19(11):1437-1448. doi:10. 1016/S1470-2045(18)30739-3

29. Bartley AN, Washington MK, Colasacco C, et al. HER2 testing and clinical decision making in gastroesophageal adenocarcinoma: guideline from the College of American Pathologists, American Society for Clinical Pathology, and the American Society of Clinical Oncology. J Clin Oncol. 2017;35:446-464. doi:10.1200/jco.2016.69.4836

30. Van Cutsem E, Bang YJ, Feng-Yi F, et al. HER2 screening data from ToGA: targeting HER2 in gastric and gastroesophageal junction cancer. Gastric Cancer. 2015;18(3):476-484. doi:10.10 07/s10120-014-0402-y

31. Hudis CA. Trastuzumab - mechanism of action and use in clinical practice. $N$ Engl J Med. 2007;357:39-51. doi:10.1056/NEJMra 043186

32. Bang YJ, Van Cutsem E, Feyereislova A, et al. Trastuzumab in combination with chemotherapy versus chemotherapy alone for treatment of HER2-positive advanced gastric or gastrooesophageal junction cancer (ToGA): a phase 3, open-label, randomised controlled trial. Lancet. 2010;376:687-697. doi:10.1016/s01406736(10)61121-x

33. Bang YJ. A randomized, open-label, phase III study of lapatinib in combination with weekly paclitaxel versus weekly paclitaxel alone in the second-line treatment of HER2 amplified advanced gastric cancer (AGC) in Asian population: Tytan study. $J$ Clin Oncol. 2013;31:11. doi:10.1200/jco.2013.31.4_suppl.11

34. Thuss-Patience PC, Shah MA, Ohtsu A, et al. Trastuzumab emtansine versus taxane use for previously treated HER2-positive locally advanced or metastatic gastric or gastro-oesophageal junction adenocarcinoma (GATSBY): an international randomised, open-label, adaptive, phase $2 / 3$ study. Lancet Oncol. 2017;18:640-653. doi:10.1016/S1470-2045(17) 30111-0

35. Tabernero J, Ho PM, Shen L, et al. Pertuzumab plus trastuzumab and chemotherapy for HER2-positive metastatic gastric or gastro-oesophageal junction cancer (JACOB): final analysis of a double-blind, randomised, placebo-controlled phase 3 study. Lancet Oncol. 2018;19:1372-1384. doi:10.1016/S1470-2045(18) 30481-9

36. Hecht JR, Bang YJ, Qin SK, et al. Lapatinib in combination with capecitabine plus oxaliplatin in human epidermal growth factor receptor 2-positive advanced or metastatic gastric, esophageal, or gastroesophageal adenocarcinoma: TRIO-013/LOGiC-a randomized phase III trial. J Clin Oncol. 2016;34:443-451. doi:10.1200/JCO.2015.62.6598

37. Al-Shamsi HO, Fahmawi Y, Dahbour I, et al. Continuation of trastuzumab beyond disease progression in HER2-positive metastatic gastric cancer: the MD Anderson experience. $J$ Gastrointest Oncol. 2016;7:499-505. doi:10.21037/jgo.2016.06.16

38. Palle J, Tougeron D, Pozet A, et al. Trastuzumab beyond progression in patients with HER2-positive advanced gastric adenocarcinoma: a multicenter AGEO study. J Clin Oncol. 2017;35:94. doi:10.1200/JCO.2017.35.4_suppl.94

39. Li Q, Jiang H, Li H, et al. Efficacy of trastuzumab beyond progression in HER2 positive advanced gastric cancer: a multicenter prospective observational cohort study. Oncotarget. 2016;7:50 656-50665. doi:10.18632/oncotarget.10456 
40. Makiyama A, Sagara K, Kawada J, et al. A randomized phase II study of weekly paclitaxel _ trastuzumab in patients with HER2-positive advanced gastric or gastro-esophageal junction cancer refractory to trastuzumab combined with fluoropyrimidine and platinum: WJOG7112G (T-ACT). J Clin Oncol. 2018; 36:4011. doi:10.1200/JCO.2018.36.15_suppl.4011

41. Saeki H, Oki E, Kashiwada T, et al. Re-evaluation of HER2 status in patients with HER2-positive advanced or recurrent gastric cancer refractory to trastuzumab (KSCC1604). Eur J Cancer. 2018;105:41-49. doi:10.1016/j.ejca.2018.09.024

42. Ogitani Y, Aida T, Hagihara K, et al. DS-8201a, a novel HER2-targeting ADC with a novel DNA topoisomerase I inhibitor, demonstrates a promising antitumor efficacy with differentiation from T-DM1. Clin Cancer Res. 2016;22:5097-5108. doi:10.1158/1078-0432.CCR-15-2822

43. Shitara K, Bang YJ, Iwasa S, et al. Trastuzumab deruxtecan in previously treated HER2-positive gastric cancer. $N$ Engl $J$ Med. 2020;382(25):2419-2430. doi:10.1056/NEJMoa2004 413

44. Yu S, Park J, Kwon W, et al. Trastuzumab deruxtecan (T-DXd) sensitivity in various levels of HER2 expressing gastric cancer cells. Presented at: AACR Annual Meeting 2021; April 10-15, 2021; Virtual. Abstract 945.

45. Seo S, Park S, Ryu M, et al. Prognostic impact of fibroblast growth factor receptor 2 gene amplification in patients receiving fluoropyrimidine and platinum chemotherapy for metastatic and locally advanced unresectable gastric cancers. Oncotarget. 2017;8 (20):33844-33854. doi:10.18632/oncotarget.12953

46. Han N, Kim MA, Lee HS, Kim WH. Evaluation of fibroblast growth factor receptor 2 expression, heterogeneity and clinical significance in gastric cancer. Pathobiology. 2015;82(6):269-279. doi: $10.1159 / 000441149$

47. Ahn S, Lee J, Hong M, et al. FGFR2 in gastric cancer: protein overexpression predicts gene amplification and high H-index predicts poor survival. Mod Pathol. 2016;29(9):1095-1103. doi:10.1038/modpathol.2016.96

48. Nagatsuma AK, Aizawa M, Kuwata T, et al. Expression profiles of HER2, EGFR, MET and FGFR2 in a large cohort of patients with gastric adenocarcinoma. Gastric Cancer. 2015;18 (2):227-238. doi:10.1007/s10120-014-0360-4

49. Tokunaga R, Imamura Y, Nakamura K, et al. Fibroblast growth factor receptor 2 expression, but not its genetic amplification, is associated with tumor growth and worse survival in esophagogastric junction adenocarcinoma. Oncotarget. 2016;7(15):19748. doi:10.18632/oncotarget.7782

50. Wainberg ZA, Enzinger PC, Kang YK, et al. Randomized double-blind placebo-controlled phase 2 study of bemarituzumab combined with modified FOLFOX6 (mFOLFOX6) in first-line (1L) treatment of advanced gastric/gastroesophageal junction adenocarcinoma (FIGHT). J Clin Oncol. 2021;39(3_suppl):160. doi:10.1200/JCO.2021.39.3_suppl.160

51. Kowanetz M, Ferrara N. Vascular endothelial growth factor signaling pathways: therapeutic perspective. Clin Cancer Res. 2006;12:5018-5022. doi:10.1158/1078-0432.CCR-06-1520

52. Tanaka T, Ishiguro H, Kuwabara $\mathrm{Y}$, et al. Vascular endothelial growth factor C (VEGF-C) in esophageal cancer correlates with lymph node metastasis and poor patient prognosis. J Exp Clin Cancer Res. 2010;29:83. doi:10.1186/1756-9966-29-83

53. Omoto I, Matsumoto M, Okumura H, et al. Expression of vascular endothelial growth factor-C and vascular endothelial growth factor receptor-3 in esophageal squamous cell carcinoma. Oncol Lett. 2014;7:1027-1032. doi:10.3892/ol.2014.1823

54. Ferrara N, Hillan KJ, Novotny W. Bevacizumab (Avastin), a humanized anti-VEGF monoclonal antibody for cancer therapy. Biochem Biophys Res Commun. 2005;333:328-335. doi:10.1016/j.bbrc.2005.05.132
55. Van Cutsem E, de Haas S, Kang Y-K, et al. Bevacizumab in combination with chemotherapy as first-line therapy in advanced gastric cancer: a biomarker evaluation from the AVAGAST randomized phase III trial. J Clin Oncol. 2012;30:2119-2127. doi:10.1200/jco.2011.39.9824

56. Lorenzen S, Thuss-Patience PC, Pauligk C, et al. FOLFIRI plus ramucirumab versus paclitaxel plus ramucirumab as second-line therapy for patients with advanced or metastatic gastroesophageal adenocarcinoma with or without prior docetaxel- results from the phase II RAMIRIS Study of the AIO. ASCO Abstr. 2020;38 (15):4514. doi:10.1200/JCO.2020.38.15_suppl.4514

57. Klempner SJ, Maron SB, Chase L, et al. Initial report of second-line FOLFIRI in combination with ramucirumab in advanced gastroesophageal adenocarcinomas: a multi-institutional retrospective analysis. Oncologist. 2019;24(4):475-482. doi:10.1634/theoncologist.20180602. PMID: 30470690; PMCID: PMC6459251.

58. Kelly RJ. Immunotherapy for esophageal and gastric cancer. $A m$ Soc Clin Oncol Educ Book. 2017;37:292-300. doi:10.1200/ EDBK_175231

59. Ahn E, Araki K, Hashimoto M, et al. Role of PD-1 during effector CD8 T cell differentiation. Proc Natl Acad Sci USA. 2018;115:4749-4754. doi:10.1073/pnas.1718217115

60. Fuchs CS, Doi T, Jang RW, et al. Safety and efficacy of pembrolizumab monotherapy in patients with previously treated advanced gastric and gastroesophageal junction cancer: phase 2 clinical KEYNOTE-059 trial. JAMA Oncol. 2018;4(5):e180013. doi:10.1001/jamaoncol.2018.0013

61. Kang YK, Boku N, Satoh T, et al. Nivolumab in patients with advanced gastric or gastro-oesophageal junction cancer refractory to, or intolerant of, at least two previous chemotherapy regimens (ONO-4538-12, ATTRACTION-2): a randomised, double-blind, placebo-controlled, phase 3 trial. Lancet. 2017;390:2461-2471. doi:10.1016/S0140-6736(17)31827-5

62. Bang YJ, Ruiz EY, Van Cutsem E, et al. Phase III, randomised trial of avelumab versus physician's choice of chemotherapy as third-line treatment of patients with advanced gastric or gastro-oesophageal junction cancer: primary analysis of JAVELIN gastric 300. Ann Oncol. 2018;29:2052-2060. doi:10.1093/annonc/mdy264

63. Kojima T, Muro K, Francois E, et al. Pembrolizumab versus chemotherapy as second-line therapy for advanced esophageal cancer: the phase 3 KEYNOTE-181 study. 2019 Gastrointestinal Cancers Symposium; Presented January 17, 2019; Abstract 2.

64. Kato K, Cho BC, Takahashi M, et al. Nivolumab versus chemotherapy in patients with advanced oesophageal squamous cell carcinoma refractory or intolerant to previous chemotherapy (ATTRACTION-3): a multicentre, randomised, open-label, phase 3 trial. Lancet Oncol. 2019;20:1506-1517. doi:10.1016/ S1470-2045(19)30626-6

65. Huang J, Xu J, Chen Y, et al. Camrelizumab versus investigator's choice of chemotherapy as second-line therapy for advanced or metastatic oesophageal squamous cell carcinoma (ESCORT): a multicentre, randomised, open-label, phase 3 study. Lancet Oncol. 2020;21(6):832-842. doi:10.1016/S1470-2045(20)30110-8

66. BeiGene, Ltd. BeiGene announces positive topline results for global phase 3 trial of tislelizumab in esophageal squamous cell carcinoma; 2021. Available from: http://bit.ly/39qV6Hn. Accessed January 28, 2021.

67. Kojima T, Shah MA, Muro K, et al. Randomized phase III KEYNOTE-181 study of pembrolizumab versus chemotherapy in advanced esophageal cancer. $J$ Clin Oncol. 2020;38 (35):4138-4148. doi:10.1200/JCO.20.01888. PMID: 33026938.

68. Shitara K, Özgüroğlu M, Bang YJ, et al. Pembrolizumab versus paclitaxel for previously treated, advanced gastric or gastro-oesophageal junction cancer (KEYNOTE-061): a randomised, open-label, controlled, phase 3 trial. Lancet. 2018;392(10142):123-133. doi:10.1016/S0140-6736(18)31257-1 
69. Nakajima TE, Kadowaki S, Minashi K, et al. Multicenter phase I/ II study of nivolumab combined with paclitaxel plus ramucirumab as second-line treatment in patients with advanced gastric cancer. Clin Cancer Res. 2021;27:1029-1036. doi:10.1158/10780432.CCR-20-3559

70. Moehler M, Dvorkin M, Boku N, et al. Phase III trial of avelumab maintenance after first-line induction chemotherapy versus continuation of chemotherapy in patients with gastric cancers: results from JAVELIN gastric 100. J Clin Oncol. 2020;39:JCO-20.

71. Shitara K, Van Cutsem E, Bang Y, et al. Efficacy and safety of pembrolizumab or pembrolizumab plus chemotherapy vs chemotherapy alone for patients with first-line, advanced gastric cancer: the KEYNOTE-062 phase 3 randomized clinical trial. JAMA Oncol. 2020;6(10):1571-1580. doi:10.1001/jamaoncol.2020.3370

72. Janjigian YY, Shitara K, Moehler M, et al. First-line nivolumab plus chemotherapy versus chemotherapy alone for advanced gastric, gastro-oesophageal junction, and oesophageal adenocarcinoma (CheckMate 649): a randomised, open-label, phase 3 trial. Lancet. 2021;398:27-40. doi:10.1016/S0140-6736(21)00797-2

73. Bristol Myers Squibb. US food and drug administration approves opdivo (nivolumab) in combination with chemotherapy for patients with advanced or metastatic gastric cancer, gastroesophageal junction cancer, and esophageal adenocarcinoma; 2021. Available from: https://news.bms.com/news/details/2021/U.S.-Food-and-DrugAdministration-Approves-Opdivo-nivolumab-in-Combination-with -Chemotherapy-for-Patients-with-Advanced-or-Metastatic-GastricCancer-Gastroesophageal-Junction-Cancer-and-EsophagealAdenocarcinoma/default.aspx. Accessed April 16, 2021.

74. Janjigian YY, Kawazoe A, Yanez PE, et al. Pembrolizumab plus trastuzumab and chemotherapy for HER2+ metastatic gastric or gastroesophageal junction (G/GEJ) cancer: initial findings of the global phase 3 KEYNOTE-811 study. J Clin Oncol. 2021;39 (15_suppl):4013. doi:10.1200/JCO.2021.39.15_suppl.4013

75. NCCN. NCCN development and update of guidelines; 2021. Available from: https://www.nccn.org/guidelines/guidelines-process /development-and-update-of-guidelines. Accessed June 24, 2021.

76. Tesniere A, Schlemmer F, Boige V, et al. Immunogenic death of colon cancer cells treated with oxaliplatin. Oncogene. 2010;29:482-491. doi:10.1038/onc.2009.356

77. Terenzi A, Pirker C, Keppler BK, Berger W. Anticancer metal drugs and immunogenic cell death. $J$ Inorg Biochem. 2016;165:71-79. doi:10.1016/j.jinorgbio.2016.06.021

78. Bezu L, Gomes-da-silva LC, Dewitte H, et al. Combinatorial strategies for the induction of immunogenic cell death. Front Immunol. 2015;6:187.

79. Martins I, Kepp O, Schlemmer F, et al. Restoration of the immunogenicity of cisplatin-induced cancer cell death by endoplasmic reticulum stress. Oncogene. 2011;30(10):1147-1158. doi:10. 1038/onc.2010.500

80. Aranda F, Bloy N, Pesquet J, et al. Immune-dependent antineoplastic effects of cisplatin plus pyridoxine in non-small-cell lung cancer. Oncogene. 2015;34(23):3053-3062. doi:10.1038/onc.20 14.234

81. Park SJ, Ye W, Xiao R, et al. Cisplatin and oxaliplatin induce similar immunogenic changes in preclinical models of head and neck cancer. Oral Oncol. 2019;95:127-135. doi:10.1016/j. oraloncology.2019.06.016

82. Boku N, Ryu MH, Oh DY, et al. Nivolumab plus chemotherapy versus chemotherapy alone in patients with previously untreated advanced or recurrent gastric/gastroesophageal junction (G/GEJ) cancer: ATTRACTION-4 (ONO-4538-37) study. Ann Oncol. 2020;31:1142-1215. doi:10.1016/j.annonc.2020.08.2297

83. Lei M, Siemers NO, Pandya D, et al. Analyses of PD-L1 and inflammatory gene expression association with efficacy of nivolumab \pm ipilimumab in gastric cancer/gastroesophageal junction cancer. Clin Cancer Res. 2021;27(14):3926-3935.
84. Kato K, Sun JM, Shah MA, et al. Pembrolizumab plus chemotherapy versus chemotherapy as first-line therapy in patients with advanced esophageal cancer: the phase 3 KEYNOTE-590 study. Ann Oncol. 2020;31:S1192-S1193. doi:10.1016/j.annonc. 2020.08.2298

85. FDA. FDA approves pembrolizumab for esophageal or GEJ carcinoma; 2021. Available from: https://bit.ly/3191ONi. Accessed March 22, 2021.

86. FDA. Meeting of the oncologic drugs advisory committee meeting announcement; 2021. Available from: https:/www.fda.gov/ advisory-committees/advisory-committee-calendar/april-27-292021-meeting-oncologic-drugs-advisory-committee-meetingannouncement-04272021-04292021. Accessed April 29, 2021.

87. $\mathrm{Xu} \mathrm{RH}$, Luo H, Lu J, et al. ESCORT-1st: a randomized, double-blind, placebo-controlled, phase 3 trial of camrelizumab plus chemotherapy vs chemotherapy in patients with untreated advanced or metastatic esophageal squamous cell carcinoma (ESCC). J Clin Oncol. 2021;39(suppl 15):4000. doi:10.1200/ JCO.2021.39.15_suppl.4000

88. Chau I, Doki Y, Ajani JA, et al. Nivolumab (NIVO) plus ipilimumab (IPI) or NIVO plus chemotherapy (chemo) versus chemo as first-line (1L) treatment for advanced esophageal squamous cell carcinoma (ESCC): first results of the CheckMate 648 study. J Clin Oncol. 2021;39(18_suppl):LBA4001-LBA4001. doi:10.1200/JCO.2021.39.15_suppl.LBA4001

89. Liu L, Yang Y, Burns R, et al. Margetuximab mediates greater Fc-dependent anti-tumor activities than trastuzumab or pertuzumab in vitro. Cancer Res. 2019;79(Suppl.13):Abstract 1538.

90. Nordstrom JL, Gorlatov S, Zhang W, et al. Anti-tumor activity and toxicokinetics analysis of MGAH22, an anti-HER2 monoclonal antibody with enhanced Fc $\gamma$ receptor binding properties. Breast Cancer Res. 2011;13(6):R123. doi:10.11 $86 /$ bcr3069

91. Nordstrom JL, Muth J, Erskine CL, et al. High frequency of HER2-specific immunity observed in patients (pts) with HER2 + cancers treated with margetuximab (M), an Fc-enhanced anti-HER2 monoclonal antibody (mAb). J Clin Oncol. 2019;37(Suppl.15):Abstract 1030. doi:10.1200/JCO.2019.37. 15_suppl.1030

92. Catenacci DV, Kang YK, Park H, et al. Margetuximab plus pembrolizumab in patients with previously treated, HER2-positive gastro-oesophageal phase 1b-2 trial. Lancet Oncol. 2020;2045:1-11.

93. Catenacci DV, Rosales M, Chung HC, et al. MAHOGANY: margetuximab combination in HER2+ unresectable/metastatic gastric/gastroesophageal junction adenocarcinoma. Future Oncol. 2021;17:1155-1164. doi:10.2217/fon-2020-1007

94. Rha SY, Lee C-K, Kim HS, et al. A multi-institutional phase Ib/II trial of first-line triplet regimen (Pembrolizumab, Trastuzumab, Chemotherapy) for HER2-positive advanced gastric and gastroesophageal junction cancer (PANTHERA Trial): molecular profiling and clinical update. J Clin Oncol. 2021;39:218. doi:10.1200/ JCO.2021.39.3 suppl.218

95. Hao D, He S, Harada K, et al. Integrated genomic profiling and modelling for risk stratification in patients with advanced oesophagogastric adenocarcinoma. Gut. 2020;gutjnl-2020-322707. doi:10.1136/gutjnl-2020-322707

96. Sahin U, Türeci Ö, Manikhas G, et al. FAST: a randomised phase II study of zolbetuximab (IMAB362) plus EOX vs EOX alone for first-line treatment of advanced CLDN18. 2 positive gastric and gastro-oesophageal adenocarcinomas. Ann Oncol. 2021;32: 609-619. doi:10.1016/j.annonc.2021.02.005

97. Kato Y, Tabata K, Kimura T, et al. Lenvatinib plus anti-PD-1 antibody combination treatment activates CD8p T cells through reduction of tumor-associated macrophage and activation of the interferon pathway. PLoS One. 2019;14:e0212513. doi:10.1371/journal. pone. 0212513 
98. Lwin Z, Gomez-Roca C, Saada-Bouzid E, et al. Phase II study of Lenvatinib (len) plus pembrolizumab (pembro) in patients (pts) with previously treated advanced solid tumors. Ann Oncol. 2020;31(suppl_4):S1142-S1215. doi:10.1016/j.annonc. 2020.08.2271

99. Kawazoe A, Fukuoka S, Nakamura Y, et al. Lenvatinib plus pembrolizumab in patients with advanced gastric cancer in the first-line or second-line setting (EPOC1706): an open-label, single-arm, phase 2 trial. Lancet Oncol. 2020;21:1057-1065. doi:10.1016/S1470-2045(20)30271-0

100. Catenacci DV, Kang YK, Park H, et al. Margetuximab plus pembrolizumab in patients with previously treated, HER2positive gastro-oesophageal adenocarcinoma (CP-MGAH22-05): a single-arm, phase 1b-2 trial. Lancet Oncol. 2020;21 (8):1066-1076. doi:10.1016/S1470-2045(20)30326-0

101. Anderson AC, Joller N, Kuchroo VK. Lag-3, Tim-3, and TIGIT: co-inhibitory receptors with specialized functions in immune regulation. Immunity. 2016;44:989-1004. doi:10.1016/j. immuni.2016.05.001

102. Bristol Myers Squibb. Bristol Myers Squibb announces RELATIVITY-047, a trial evaluating anti-LAG-3 antibody relatlimab and opdivo (nivolumab) in patients with previously untreated metastatic or unresectable melanoma, meets primary endpoint of progression-free survival. News release; 2021. Available from: https://bit.ly/3ffwCnI. Accessed March 25, 2021.

103. Yang L, Wang Y, Wang H. Use of immunotherapy in the treatment of gastric cancer. Oncol Lett. 2019;18:5681-5690.

104. Beîbnowska D, Grywalska E, Nied_zwiedzka-Rystwej P, et al. CAR-T cell therapy-an overview of targets in gastric cancer. J Clin Med. 2020;9:1894. doi:10.3390/jcm9061894

105. Song Y, Tong C, Wang Y, et al. Effective and persistent antitumor activity of HER2-directed CAR-T cells against gastric cancer cells in vitro and xenotransplanted tumors in vivo. Protein Cell. 2018;9:867-878. doi:10.1007/s13238-017-0384-8

106. Maher J, Wilkie S. CAR mechanics: driving T cells into the MUC of cancer. Cancer Res. 2009;69:4559-4562. doi:10.1158/00085472.CAN-09-0564

107. ClinicalTrials.gov. Single-arm, two phase, multicenter trial to evaluating the efficacy and safety of the CAR-T for stomach cancer; 2021. Available from: https://clinicaltrials.gov/ct2/show/ NCT02725125. Accessed March 15, 2020.

108. Knödler M, Körfer J, Kunzmann V, et al. Randomised phase II trial to investigate catumaxomab (anti-EpCAM_anti-CD3) for treatment of peritoneal carcinomatosis in patients with gastric cancer. Br J Cancer. 2018;119:296-302. doi:10.1038/s41416-018-0150-6

109. Dottermusch M, Krüger S, Behrens HM, Halske C, Röcken C. Expression of the potential therapeutic target claudin-18.2 is frequently decreased in gastric cancer: results from a large Caucasian cohort study. Virchows Arch. 2019;475:563-571. doi:10.1007/s00428-019-02624-7

110. Singh P, Toom S, Huang Y. Anti-claudin 18.2 antibody as new targeted therapy for advanced gastric cancer. J Hematol Oncol. 2017;10:105. doi:10.1186/s13045-017-0473-4

111. Jiang H, Shi Z, Wang P, et al. Claudin18.2-specific chimeric antigen receptor engineered T cells for the treatment of gastric cancer. $J$ Natl Cancer Inst. 2019;111:409-418. doi:10.1093/jnci/djy134

112. PR Newswire. CARsgen therapeutics receives IND clearance from the US FDA for CT041 CLDN18.2-CAR-T cells; 2021. Available from: https://www.prnewswire.com/news-releases/cars gen-therapeutics-receives-ind-clearancefrom-the-us-fda-for-ct041 -cldn18-2-car-t-cells-301060555.html. Accessed June 6, 2020.

113. clinicaltrials.gov. Open-label, multicenter, phase $1 \mathrm{~b}$ clinical trial to evaluate the safety and efficacy of autologous claudin 18.2 chimeric antigen receptor T-cell therapy in patients with advanced gastric or pancreatic adenocarcinoma; 2021. Available from: https://clinical trials.gov/ct2/show/NCT04404595. Accessed June 6, 2020.
114. Zhan X, Wang B, Li Z, et al. Phase I trial of Claudin 18.2-specific chimeric antigen receptor $\mathrm{T}$ cells for advanced gastric and pancreatic adenocarcinoma. $J$ Clin Oncol. 2019;37:2509. doi:10.12 00/JCO.2019.37.15_suppl.2509

115. Kankeu Fonkoua LA, Chakrabarti S, Sonbol MB, et al. Outcomes on anti-VEGFR-2/paclitaxel treatment after progression on immune checkpoint inhibition in patients with metastatic gastroesophageal adenocarcinoma. Int $J$ Cancer. 2021;149:1-9. doi:10.1002/ijc.33559.

116. Yoon HH, Fuchs CS, Özgüroğlu M, et al. O-12 KEYNOTE-061: response to subsequent therapy following second-line pembrolizumab or paclitaxel in patients with advanced gastric or gastroesophageal junction adenocarcinoma. Ann Oncol. 2020;31:236. doi:10.1016/j.annonc.2020.04.065

117. Sasaki A, Kawazoe A, Eto $T$, et al. Improved efficacy of taxanes and ramucirumab combination chemotherapy after exposure to anti-PD-1 therapy in advanced gastric cancer. ESMO Open. 2020;4(Suppl 2):e000775. doi:10.1136/esmoopen-2020-000775

118. Kawazoe A, Ando T, Hosaka H, et al. Safety and activity of trifluridine/tipiracil and ramucirumab in previously treated advanced gastric cancer: an open-label, single-arm, phase 2 trial. Lancet Gastroenterol Hepatol. 2021;6(3):209-217. doi:10.1016/S2468-1253(20)30396-4

119. Zhu M, Sonbol MB, Yoon HH. Trifluridine/tipiracil plus ramucirumab in gastric cancer. Lancet Gastroenterol Hepatol. 2021;6:154-155. doi:10.1016/S2468-1253(21)00013-3

120. Chin K, Kato K, Cho BC, et al. Three-year follow-up of ATTRACTION-3: a phase III study of nivolumab (Nivo) in patients with advanced esophageal squamous cell carcinoma (ESCC) that is refractory or intolerant to previous chemotherapy. J Clin Oncol. 2021;39:204. doi:10.1200/ JCO.2021.39.3_suppl.204

121. Cho J, Kang MS, Kim KM. Epstein-barr virus-associated gastric carcinoma and specific features of the accompanying immune response. $J$ Gastric Cancer. 2016;16:1-7. doi:10.5230/ jgc.2016.16.1.1

122. Van Beek J, Zur Hausen A, Klein Kranenbarg E, et al. EBVpositive gastric adenocarcinomas: a distinct clinicopathologic entity with a low frequency of lymph node involvement. $J$ Clin Oncol. 2004;22:664-670. doi:10.1200/JCO.2004.08.061

123. Camargo MC, Murphy G, Koriyama C, et al. Determinants of Epstein-Barr virus-positive gastric cancer: an international pooled analysis. Br J Cancer. 2011;105:38-43. doi:10.1038/bjc.2011.215

124. Kim ST, Cristescu R, Bass AJ, et al. Comprehensive molecular characterization of clinical responses to PD-1 inhibition in metastatic gastric cancer. Nat Med. 2018;24:1449-1458. doi:10.1038/ s41591-018-0101-z

125. Rha SY, Lee JB, Kim HS, et al. Open label, single-arm, multi-center phase Ib/II study to evaluate the safety and efficacy of nivolumab in combination with paclitaxel in Epstein-Barr virus (EBV)-related, or microsatellite instability-high (MSI-H), or programmed cell death ligand 1 (PD-L1) positive advanced gastric cancer (AGC). Presented at: AACR Annual Meeting 2021; April 10-15, 2021; Virtual. Abstract CT159.

126. Lordick F, Kang YK, Chung HC, et al. Capecitabine and cisplatin with or without cetuximab for patients with previously untreated advanced gastric cancer (EXPAND): a randomised, open-label phase 3 trial. Lancet Oncol. 2013;14:490-499. doi:10.1016/ S1470-2045(13)70102-5

127. Waddell T, Chau I, Cunningham D, et al. Epirubicin, oxaliplatin, and capecitabine with or without panitumumab for patients with previously untreated advanced oesophagogastric cancer (REAL3): a randomised, open-label phase 3 trial. Lancet Oncol. 2013;14:481-489. doi:10.1016/S1470-2045(13)70096-2 
128. Shah MA, Bang YJ, Lordick F, et al. Effect of fluorouracil, leucovorin, and oxaliplatin with or without onartuzumab in HER2-negative, MET-positive gastroesophageal adenocarcinoma: the METGastric randomized clinical trial JAMA Oncol. 2017;3:620-627. doi:10.1001/jamaoncol.2016. 5580
129. Catenacci DVT, Tebbutt NC, Davidenko I, et al. Rilotumumab plus epirubicin, cisplatin, and capecitabine as first-line therapy in advanced MET-positive gastric or gastro-oesophageal junction cancer (RILOMET-1): a randomised, double-blind, placebo-controlled, phase 3 trial. Lancet Oncol. 2017;18:1467-1482. doi:10.1016/S1470-2045(17)30566-1

\section{Publish your work in this journal}

OncoTargets and Therapy is an international, peer-reviewed, open access journal focusing on the pathological basis of all cancers, potential targets for therapy and treatment protocols employed to improve the management of cancer patients. The journal also focuses on the impact of management programs and new therapeutic

Submit your manuscript here: https://www.dovepress.com/oncotargets-and-therapy-journal agents and protocols on patient perspectives such as quality of life, adherence and satisfaction. The manuscript management system is completely online and includes a very quick and fair peer-review system, which is all easy to use. Visit http://www.dovepress.com/ testimonials.php to read real quotes from published authors. 